\title{
Surface electroencephalography (EEG) during the acute phase of stroke to assist with diagnosis and prediction of prognosis: a scoping review
}

\author{
Lou Sutcliffe ${ }^{\dagger}$, Hannah Lumley ${ }^{* \dagger}$, Lisa Shaw, Richard Francis and Christopher I. Price
}

\begin{abstract}
Background: Stroke is a common medical emergency responsible for significant mortality and disability. Early identification improves outcomes by promoting access to time-critical treatments such as thrombectomy for large vessel occlusion (LVO), whilst accurate prognosis could inform many acute management decisions. Surface electroencephalography (EEG) shows promise for stroke identification and outcome prediction, but evaluations have varied in technology, setting, population and purpose. This scoping review aimed to summarise published literature addressing the following questions: 1. Can EEG during acute clinical assessment identify: a) Stroke versus non-stroke mimic conditions. b) Ischaemic versus haemorrhagic stroke. c) Ischaemic stroke due to LVO. 2. Can these states be identified if EEG is applied $<6 \mathrm{~h}$ since onset. 3. Does EEG during acute assessment predict clinical recovery following confirmed stroke.

Methods: We performed a systematic search of five bibliographic databases ending 19/10/2020. Two reviewers assessed eligibility of articles describing diagnostic and/or prognostic EEG application $<72 \mathrm{~h}$ since suspected or confirmed stroke.

Results: From 5892 abstracts, 210 full text articles were screened and 39 retained. Studies were small and heterogeneous. Amongst 21 reports of diagnostic data, consistent associations were reported between stroke, greater delta power, reduced alpha/beta power, corresponding ratios and greater brain asymmetry. When reported, the area under the curve (AUC) was at least good (0.81-1.00). Only one study combined clinical and EEG data (AUC 0.88). There was little data found describing whether EEG could identify ischaemic versus haemorrhagic stroke. Radiological changes suggestive of LVO were also associated with increased slow and decreased fast waves. The only study with angiographic proof of LVO reported AUC 0.86 for detection $<24$ h since onset. Amongst 26 reports of prognostic data, increased slow and reduced fast wave EEG changes were associated with future dependency, neurological impairment, mortality and poor cognition, but there was little evidence that EEG enhanced outcome prediction relative to clinical and/or radiological variables. Only one study focussed solely on patients $<6 \mathrm{~h}$ since onset for predicting neurological prognosis post-thrombolysis, with more favourable outcomes associated with greater hemispheric symmetry and a greater ratio of fast to slow waves.
\end{abstract}

\footnotetext{
*Correspondence: hannah.lumley@ncl.ac.uk

†Lou Sutcliffe and Hannah Lumley Joint lead authors.

Stroke Research Group, Population Health Science Institute, Newcastle

University, Newcastle-Upon-Tyne, UK
}

(c) The Author(s) 2022. Open Access This article is licensed under a Creative Commons Attribution 4.0 International License, which permits use, sharing, adaptation, distribution and reproduction in any medium or format, as long as you give appropriate credit to the original author(s) and the source, provide a link to the Creative Commons licence, and indicate if changes were made. The images or other third party material in this article are included in the article's Creative Commons licence, unless indicated otherwise in a credit line to the material. If material is not included in the article's Creative Commons licence and your intended use is not permitted by statutory regulation or exceeds the permitted use, you will need to obtain permission directly from the copyright holder. To view a copy of this licence, visit http://creativecommons.org/licenses/by/4.0/. The Creative Commons Public Domain Dedication waiver (http://creativeco mmons.org/publicdomain/zero/1.0/) applies to the data made available in this article, unless otherwise stated in a credit line to the data. 
Conclusions: Although studies report important associations with EEG biomarkers, further technological development and adequately powered real-world studies are required before recommendations can be made regarding application during acute stroke assessment.

Keywords: Electroencephalography, Acute stroke, Diagnosis, Prognosis, Large vessel occlusion

\section{Background}

Stroke is responsible for a high disability, mortality and economic burden worldwide. Emergency treatments can improve outcomes [1, 2], particularly intravenous thrombolysis and mechanical thrombectomy for selected patients with ischaemic stroke. These highly timesensitive treatments reduce long-term disability when administered to appropriate patients, but urgent clinical assessment including brain Computed Tomography (CT) or Magnetic Resonance Imaging (MRI) must first determine eligibility. For mechanical thrombectomy, additional angiography (CTA or MRA) is needed to confirm the presence of large vessel occlusion (LVO), with subsequent transfer of treatable patients if they are not already at a comprehensive stroke centre [3]. Earlier identification of individual patients most likely to benefit from specific emergency treatments will improve outcomes, especially if this is possible in the prehospital setting so that ambulance admissions can be directed to the most appropriate facility.

Accurate initial identification of stroke patients is complicated by 'mimic' conditions that produce the same symptoms as stroke, such as epileptic seizures, migraine and infections. A literature review of 79 studies reported that, despite routine use of symptom checklists like the Face Arm Speech Test, an average of 27\% (range: 4-43\%) prehospital suspected stroke admissions and 10\% (range: 1-25\%) thrombolysis patients were later re-categorised as stroke mimics [4]. More complex symptom checklists have been developed to identify LVO, but these have not been widely adopted due to the unfavourable balance between specificity and sensitivity [5, 6]. Point-of-care tests to distinguish stroke from mimic patients, haemorrhagic from ischaemic stroke and/or identify LVO would allow earlier access to appropriate emergency care. Although prehospital CT brain imaging in mobile stroke units has been implemented within highly resourced healthcare systems [7], there are no other diagnostic technologies currently available with acceptable accuracy [8]. Similarly, portable technologies providing early information about prognosis could assist clinicians whilst making a range of acute management decisions, such as whether treatment of early complications would be likely to influence recovery or might possibly be futile.

Electroencephalography (EEG) is a non-invasive clinical tool frequently used in hospital-based diagnosis and management of seizures, but has also been evaluated for stroke identification and prognostication. An increase in slow-wave (delta) versus faster (alpha/beta) activity has long been recognised following a recent stroke, although the exact mechanism is uncertain [9-11]. Quantitative EEG (qEEG) has been used as a biomarker to predict outcomes in ischaemic stroke in acute and sub-acute settings $[12,13]$. Its ability to detect and size lesions $[14,15]$ suggests that it could be used as a diagnostic tool and a clinical decision aid during treatment decisions. Advances in qEEG analysis methods and algorithms such as the Brain Symmetry Index [16], and introduction of portable systems using a minimal number of electrodes $[17,18]$, have increased the practical potential for use in emergency department (ED) and pre-hospital settings [19]. We undertook a literature review to describe the use of EEG during the acute phase of stroke for stratification of unselected patients into important clinical groups, and as an aid for clinical decision-making through early estimation of prognosis. A scoping review approach was applied due to significant heterogeneity in technology and setting in this emerging field.

\section{Methods}

The Preferred Reporting Systems for Systematic Reviews and Meta-Analyses Extension for Scoping Reviews (PRISMA-ScR) framework was applied [20].

\section{Aim}

The aim was to report evidence describing the capability of EEG technologies for stratification (identification and prognostication) when applied within $72 \mathrm{~h}$ of stroke symptom onset.

\section{Objectives}

By classifying and describing clinical studies of EEG technologies applied soon after stroke symptom onset $(<72 \mathrm{~h})$, we addressed the following questions:

1. Can EEG during acute clinical assessment identify:

a) Stroke versus non-stroke mimic conditions.

b) Ischaemic versus haemorrhagic stroke.

c) Ischaemic stroke due to LVO. 
2. Can these states be identified if EEG is applied $<6 \mathrm{~h}$ of symptom onset.

3. Does EEG during acute assessment predict clinical recovery following confirmed stroke.

\section{Search strategy}

Following exploratory searches, a systematic strategy combining $\mathrm{MeSH} / \mathrm{Web}$ of Science categories and keywords was developed and executed in Ovid (selecting Medline, Embase and PsycINFO databases), Web of Science and Scopus databases up until the $19^{\text {th }}$ October 2020 inclusive. Hand searching of reference lists and citation searches of included studies were undertaken. Only published peer-reviewed literature was retained, including conference abstracts if there was sufficient information reported, but case studies were excluded. It was not necessary to contact the authors of any articles for clarification. The search strategies are listed under 'Supplement $A^{\prime}$ in the Supplementary Material.

\section{Study inclusion criteria}

Research studies and review articles, including feasibility and pilot studies, with abstracts published in English from any country were eligible for inclusion if they presented original data and appropriate statistical comparison describing the application of EEG technology for stroke identification or prognosis. It was necessary for the test population to include patients with suspected or confirmed stroke, where the EEG technique was commenced (but not necessarily completed) within $72 \mathrm{~h}$. Although this time window extended beyond the interval for delivery of emergency stroke treatments, it enabled inclusion of information from studies with a range of onset to EEG times. Studies that focused mainly or solely on seizures (including prediction of post-stroke epilepsy) or Transient Ischaemic Attack (TIA) (stroke symptoms resolved within $24 \mathrm{~h}$ ) were excluded.

Any EEG-based assessment was permissible, including but not limited to: qualitative visual analysis of EEG, qEEG, continuous EEG monitoring, the Brain Symmetry Index (BSI) and frequency-specific power measures such as delta/alpha power ratio (DAR) or (delta + theta)/ (alpha + beta) power ratio (DTABR). The study setting could be in hospital or in an ambulance, including situations where patients were conveyed to a specialist laboratory from hospital for EEG recording.

Any diagnostic process was accepted for the stroke reference standard i.e. MRI/A, CT/A and/or specialist opinion. Comparisons against mimic conditions and nonstroke/healthy controls were included when the origin of the source data was stated. However, studies were not included if stroke patient data were being compared only to standard definitions of 'healthy/normal' EEG parameters, without description of a reference data source.

Studies examining detection of LVO were included if there was direct evidence of large artery occlusion (e.g. CT angiography) or, because not many studies were expected to use this reference standard, we also considered studies reporting indirectly associated radiological features (e.g. large infarct size).

For prognostic studies we included those using any previously described clinical stroke outcome measure, or survival/death. For these studies, we reported only the main outcome of interest as stated by the authors.

\section{Study selection}

Duplicate articles were excluded. Two members of the study team $(\mathrm{LSu}+\mathrm{RF})$ reviewed titles and abstracts and selected full text articles to confirm inclusion with arbitration by a third reviewer if required (CP and/or LSh). Templates for review, extraction and quality assessment can be found under 'Supplement B' in the Supplementary Material.

\section{Data extraction}

Data were independently extracted by two reviewers ( $\mathrm{LSu}$ and $\mathrm{HL}$ ), with discrepancies resolved via group discussion.

A data extraction framework was developed and piloted by the reviewers before use, which included fields for: Year of publication, country of origin, study aims, study design, setting, inclusion/exclusion criteria, EEG technology, EEG data processing methodology, reference standard information, outcome measures, blinding, sample size, time from stroke onset to first EEG measure, major findings (including statistical significance and diagnostic accuracy) and whether all patients were represented in the data with any exclusions explained.

To assess study quality, a simple scoring system (0-5) was created which reflected the main indicators of good research design i.e. clear eligibility criteria; clearly defined technology; clearly defined reference standard and/or outcome measure; blinding; whether all participants were accounted for in the results presented. Studies were not excluded based on quality, but quality and design were considered during recommendations based upon strength of evidence.

\section{Data synthesis}

As this was a scoping review, there was no a-priori plan for data meta-analysis and a narrative description is provided. Data are presented in tables according to reference 


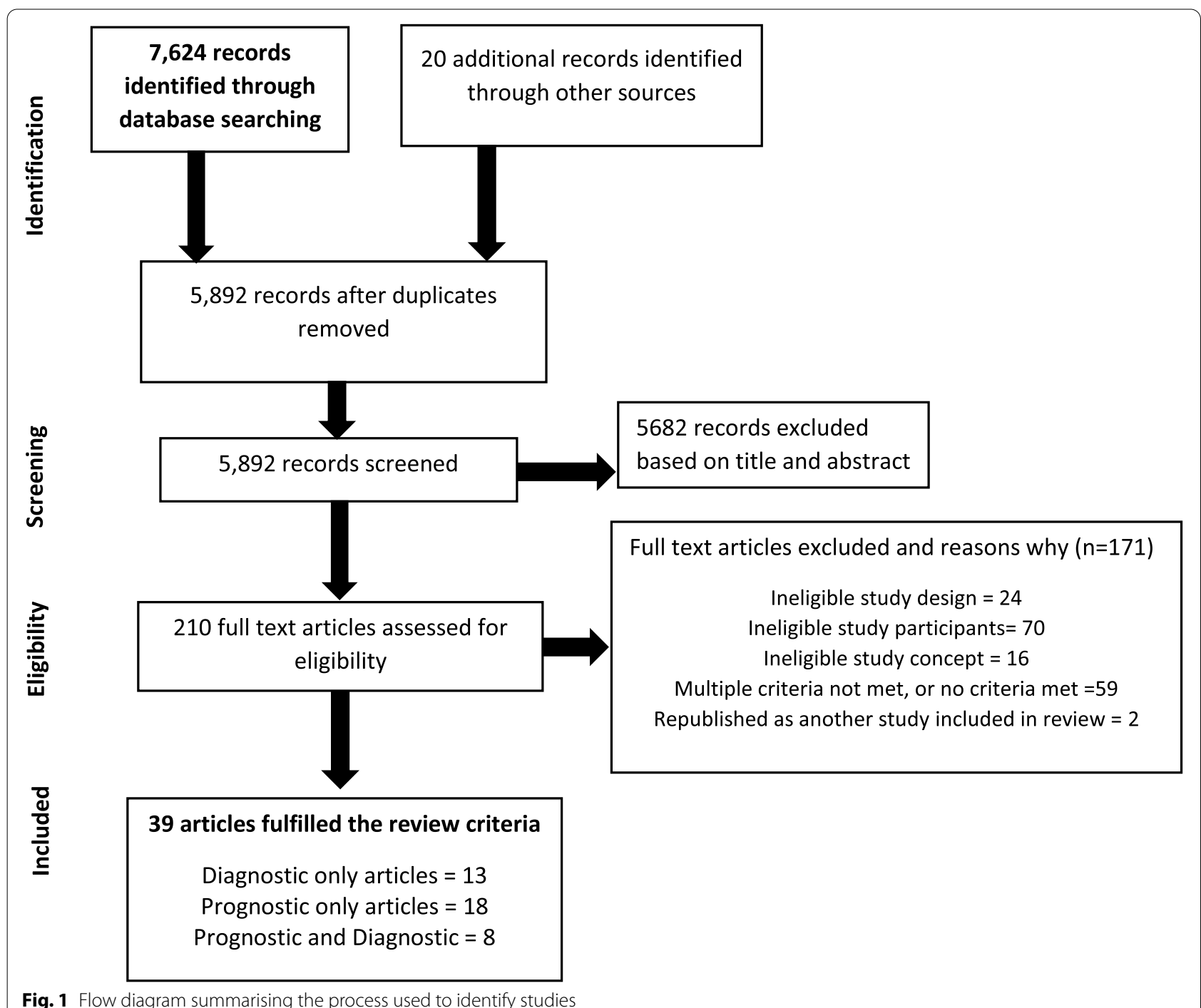

Fig. 1 Flow diagram summarising the process used to identify studies

standard or outcome measure in ascending order of publication date.

\section{Results}

Databases searches identified 7624 articles, with 20 more from hand-searching relevant review publications. After removal of duplicates, 5892 abstracts remained. Of these, 5682 abstracts did not meet the inclusion criteria. The remaining 210 full text articles were assessed (Fig. 1) and 171 articles were excluded: 24 did not meet study design criterion, 70 did not meet the participants criterion, 16 did not address the review question, 59 did not meet multiple criteria, and 2 were republished as another included study. After full text review, 39 articles were included for data extraction and quality assessment: 13 reporting diagnostic data only, 18 reporting prognostic data only and eight articles reporting both.

\section{Included studies}

Study designs were diverse. The majority were cohort $(n=24)$ or case-control $(n=14)$ studies, although very few specifically used these terms. Only one study [21] was considered a true diagnostic accuracy study, as the investigators performing the EEG were blinded to patients' clinical status and the reference standard was determined in advance (clinical specialist opinion).

\section{Population}

There was a wide geographic distribution of studies: Eight in China; seven in Australia; five in USA; three each in Belgium and Cuba; two each in Portugal and Israel and one each in Germany, Indonesia, Ukraine, Italy, Brazil, Finland, France and Hungary. Nationality was unclear for one conference abstract. Apart from one study where the 
setting was unclear, most were conducted in acute care settings in hospital (two in Emergency Departments; four in an Intensive Care Unit; seven in a neurology department; 10 in a stroke unit and 15 in hospital with no clear department). No studies were conducted in an ambulance or in the pre-hospital setting.

The median number of patients across the 39 articles was 33 (range $=11-199$ ). Inclusion and exclusion criteria were extremely variable, with some studies requiring extensive lists of exclusions and others giving limited or no information beyond a diagnosis of "stroke". Two diagnostic studies $[22,23]$ included TIA as part of the stroke patient sample, whereas others excluded TIA. Inclusion/ exclusion criteria that appeared frequently are listed in Supplement C (Table S1) in the Supplementary Material.

Median time from stroke onset to EEG application was $48 \mathrm{~h}$ (range $=4.5-72 \mathrm{~h}$ ) when this information was available. There was only one study where all patients were within six hours of symptoms onset, which was examining EEG indicators for recovery of neurological impairment after thrombolysis [24].

\section{EEG techniques}

Most studies used quantitative EEG measures as their stroke biomarkers $(n=30)$. A smaller number used either topographic EEG mapping $(n=4)$ or qualitative analysis of abnormal EEG patterns such as epileptiform activity based on expert assessment $(n=13)$. Eight studies used multiple EEG markers. A single study made use of a deep learning neural network to select optimal diagnostic EEG conditions [23]. All studies that reported electrode locations used the international $10 / 20$ system $(n=36)$. Characteristics of EEG techniques commonly specified by included studies are listed in Supplement C (Table S2) in the Supplementary Material.

\section{Reference standards and outcome measures}

Amongst 21 articles reporting diagnostic information, the most common reference standard was "specialist opinion including imaging" $(n=11)$. Five articles used 'specialist opinion' without providing further detail. Five articles used CT or MRI, one study used CT and one study used MRI alone. In 12 studies the reference standard assessment was performed within $72 \mathrm{~h}$ or during the inpatient stay. In three more, it was additionally recorded over a longer time-window (up to $118 \mathrm{~h}$ ), and in six there was no time reported.

Amongst 26 studies reporting prognostic information, the most frequent outcome measure was an assessment of dependency $(n=12)$ including modified Rankin Score (mRS), Glasgow Outcome Scale (GOS), and Barthel Index (BI). Eight studies assessed neurological outcome using the National Institutes of
Health Stroke Scale (NIHSS). Smaller numbers of articles assessed cognitive impairment via the Montreal Cognitive Assessment (MoCA) or a dementia diagnosis $(n=4)$, and survival/mortality $(n=2)$. Most studies assessed the outcome measure after discharge or more than $72 \mathrm{~h}$ after stroke, at a time point ranging from seven days to seven years.

\section{Quality of studies}

Only two articles showed evidence of a sample size calculation $[25,26]$. One other article included a post-hoc power calculation and ascertained that only some of their EEG parameters/sub-analyses had adequate statistical power [15].

Sixteen articles had some evidence of outcome blinding. It was stated that the EEG assessor was blinded to clinical data in the reference standard for three diagnostic articles, but there was no explicit indication that the clinician assessing the reference standard was blinded to EEG data. Eight articles with a prognostic aim reported a variety of blinding methods: EEG and outcome assessors blinded $(n=5)$, only EEG assessor blinded $(n=3)$, only outcome assessor blinded $(n=1)$ and patients blinded $(n=1)$. For three articles that had both diagnostic and prognostic aims and any form of blinding, there was evidence that the EEG assessor (or secondary EEG assessor) or outcome assessor was blinded to clinical data.

There were five articles where it was not possible to account for all the participants due to unclear text, figures, or presentation of data representing only individual patients.

Of the 21 articles with a diagnostic aim, 15 had evidence of a predetermined reference standard including specialist opinion. Of the 26 articles with a prognostic aim, the outcome measure was clearly defined for five studies but the majority were unclear as to whether a measure had been selected before commencing recruitment.

\section{Study data \\ 1a) Identification of stroke versus non-stroke}

Seventeen articles considered whether EEG could distinguish stroke from non-stroke; two of which specifically aimed to distinguish between stroke and TIA. Studies are summarised in Table 1, grouped by year of publication and reference standard.

Fifteen articles examined differences between stroke from healthy controls, or an identified healthy control dataset, and two compared stroke with stroke mimic conditions [22, 23]. Median article quality score was 3 (range 2-5), but even higher quality reports included only modest numbers of patients (e.g. ischaemic stroke cases ranged from 6-65 patients). 


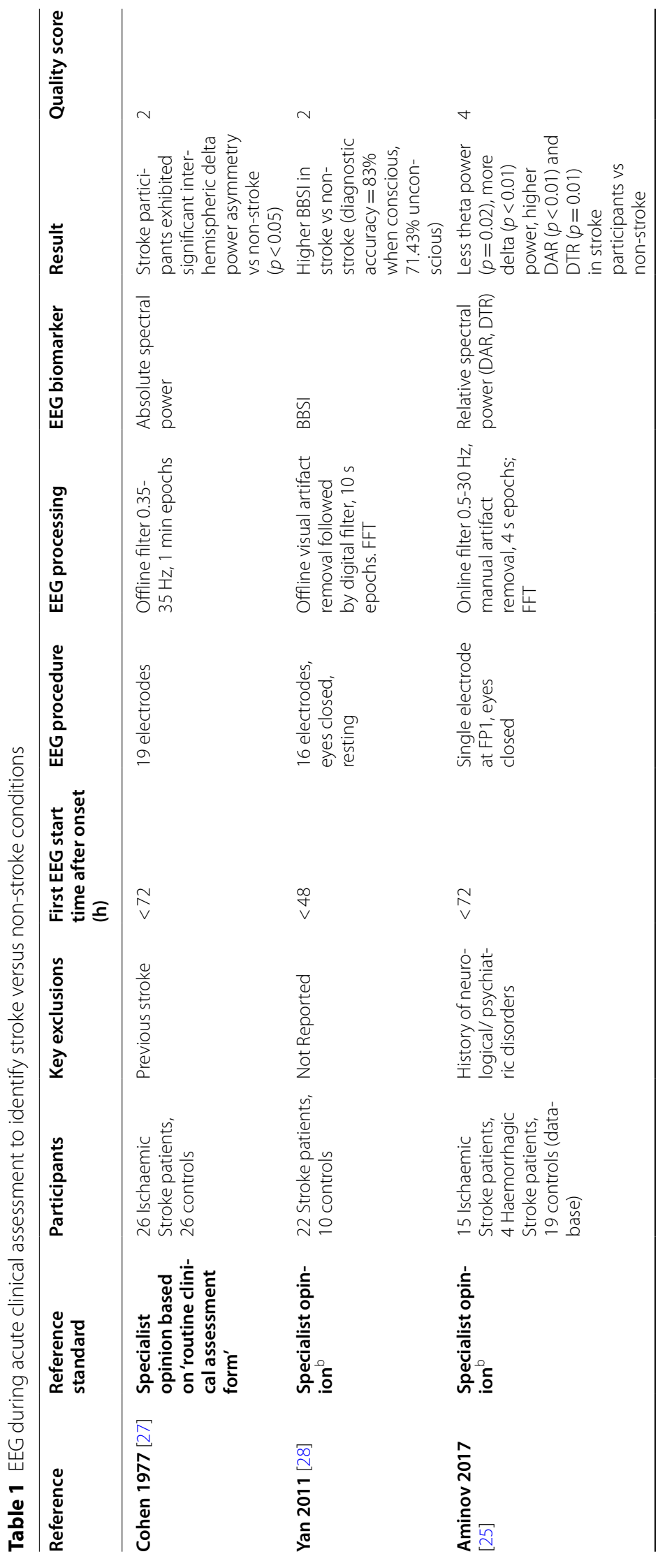




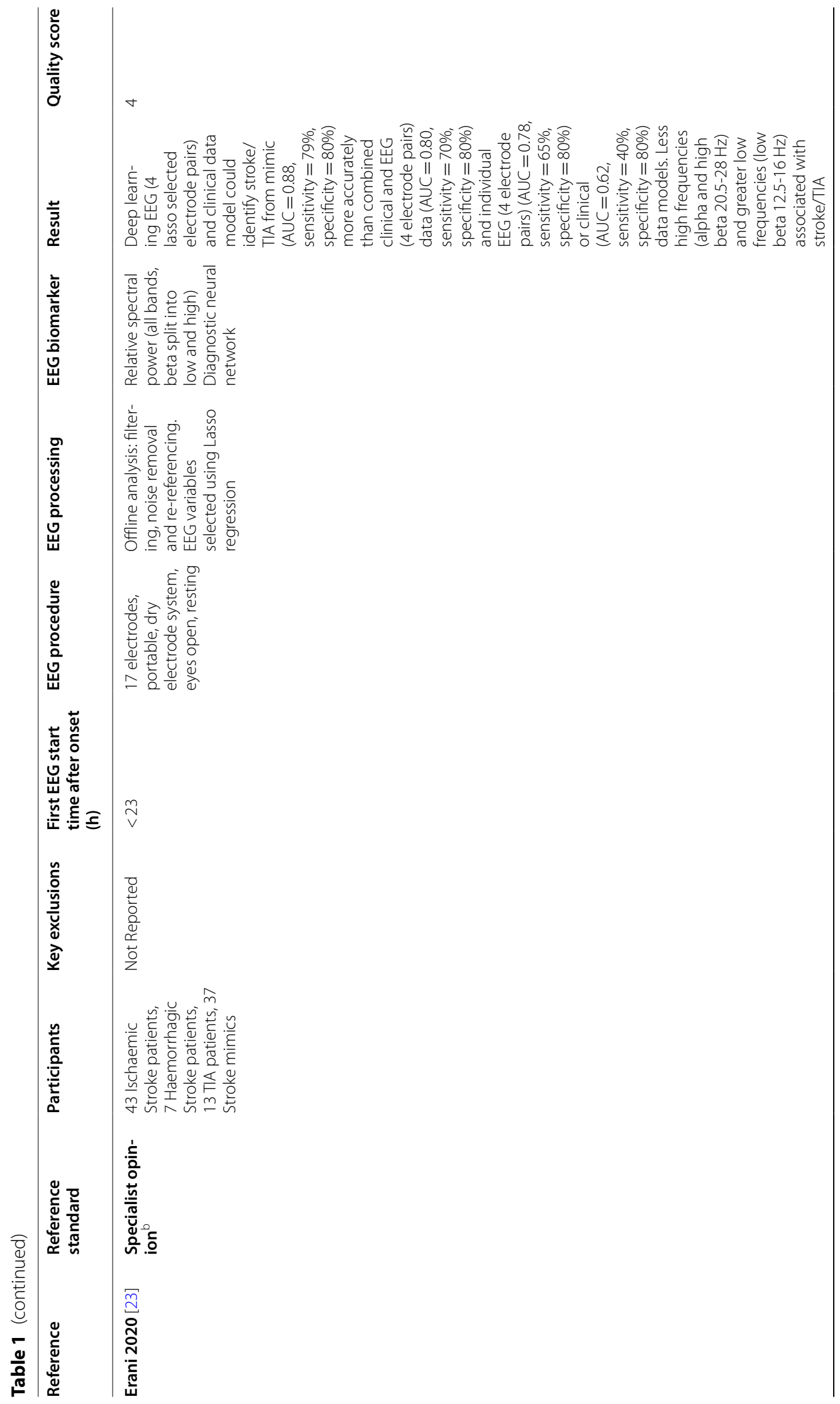




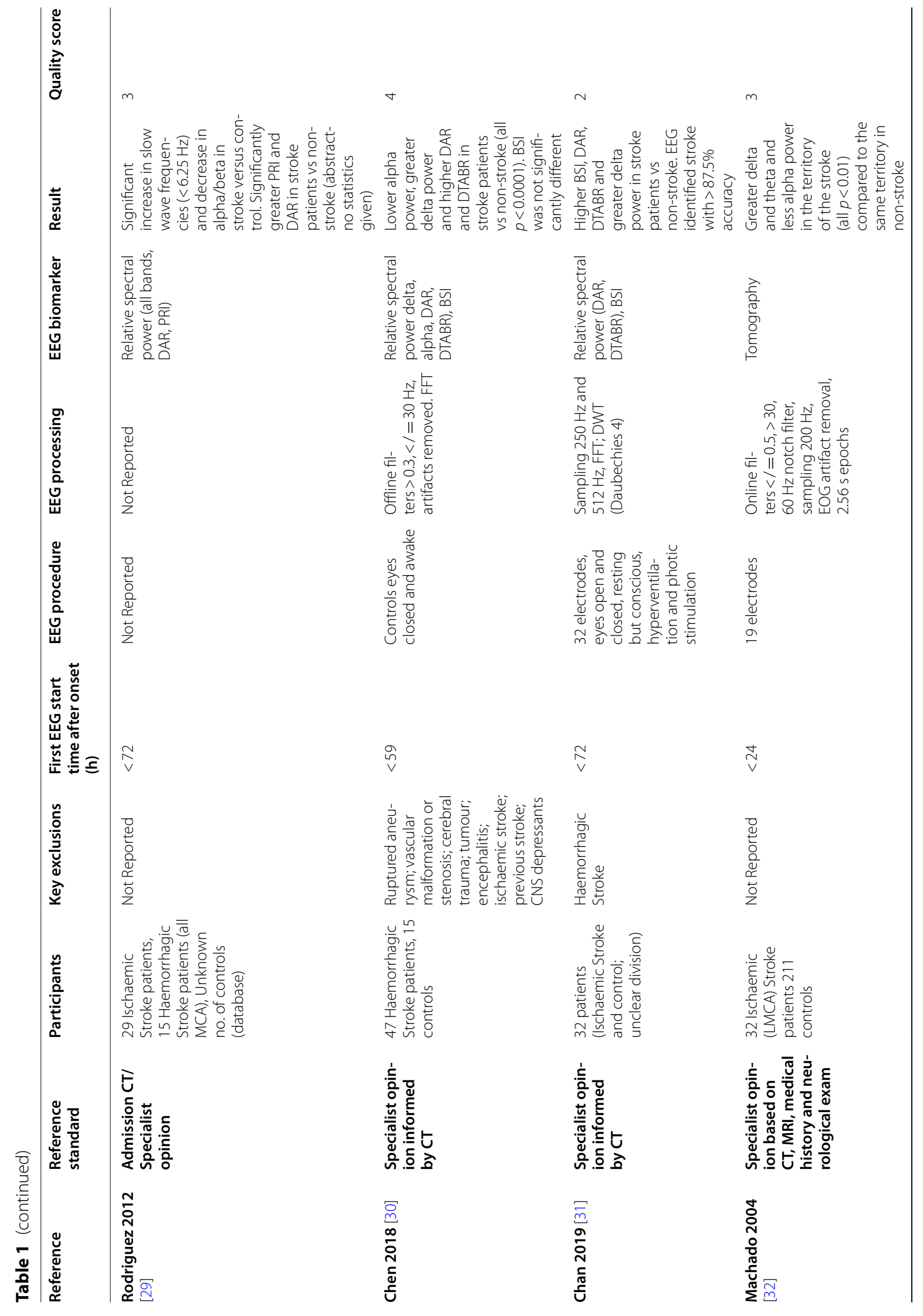




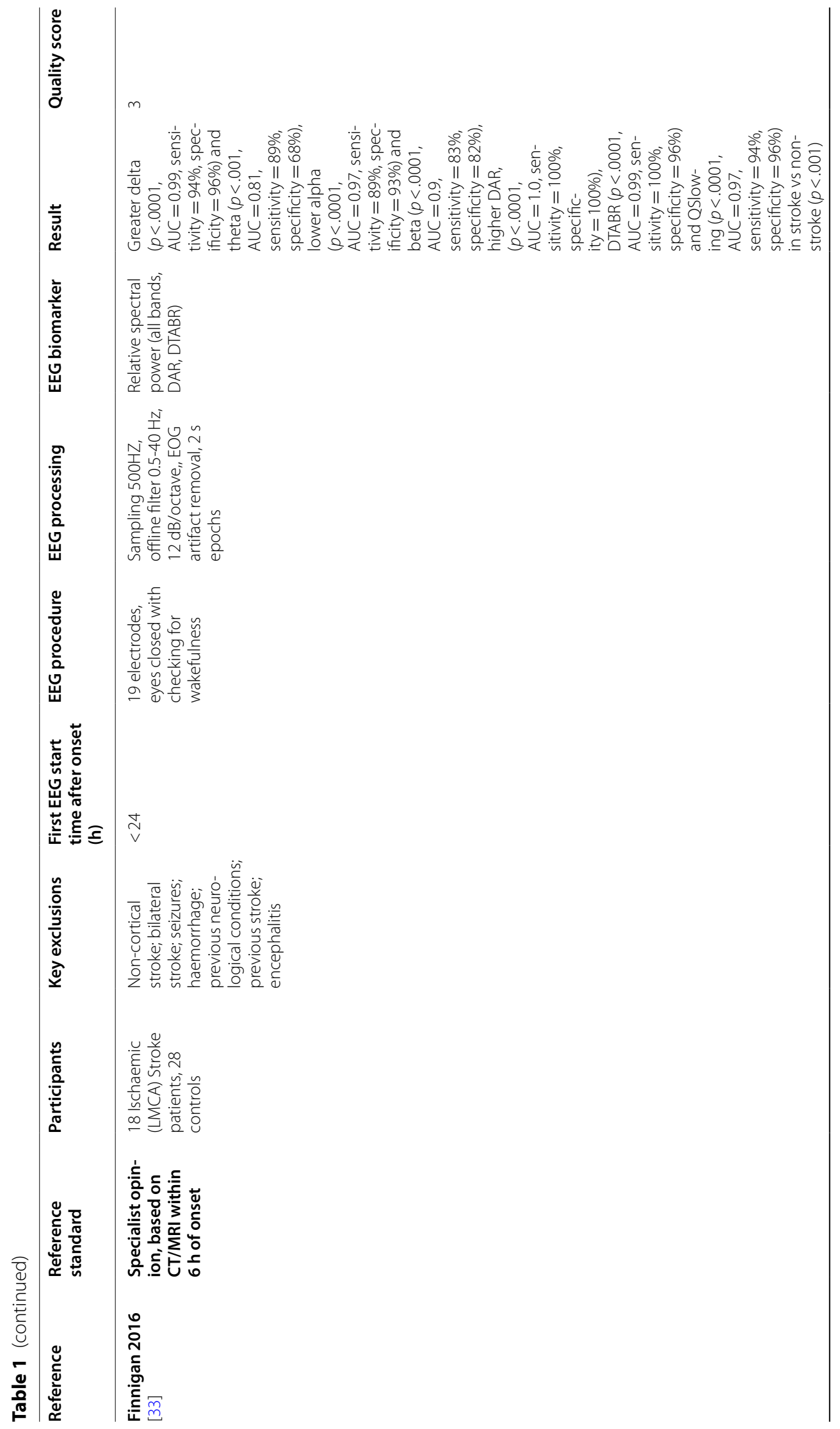




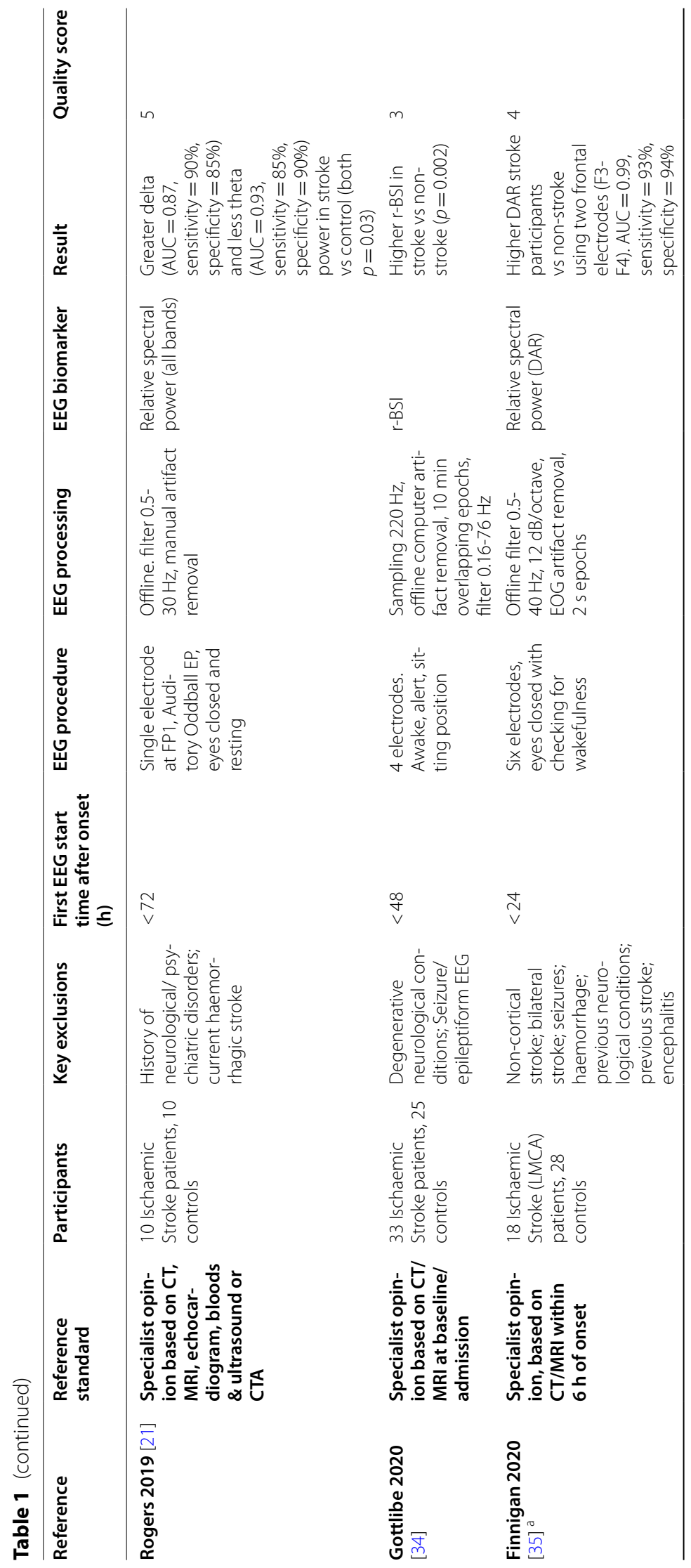




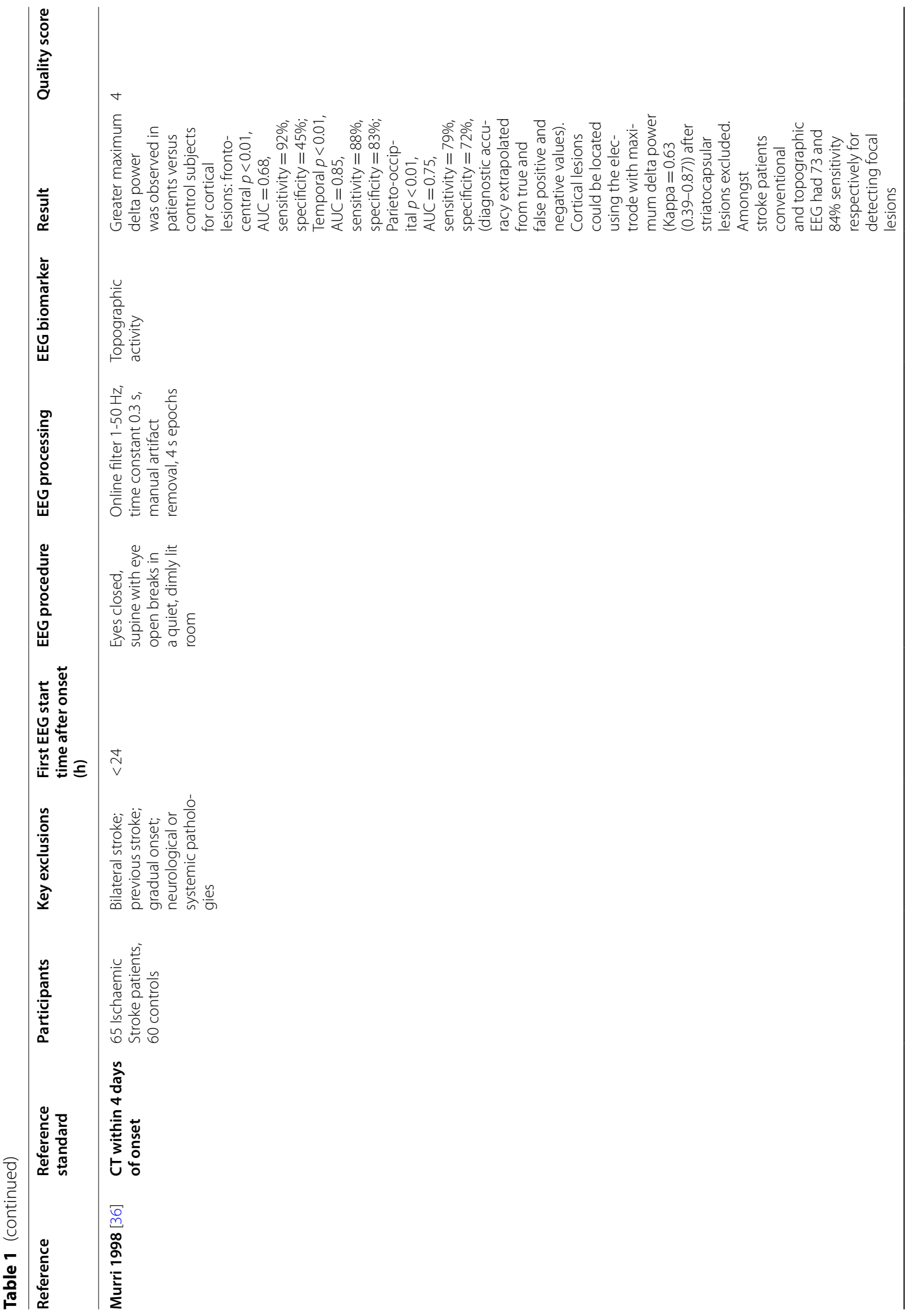




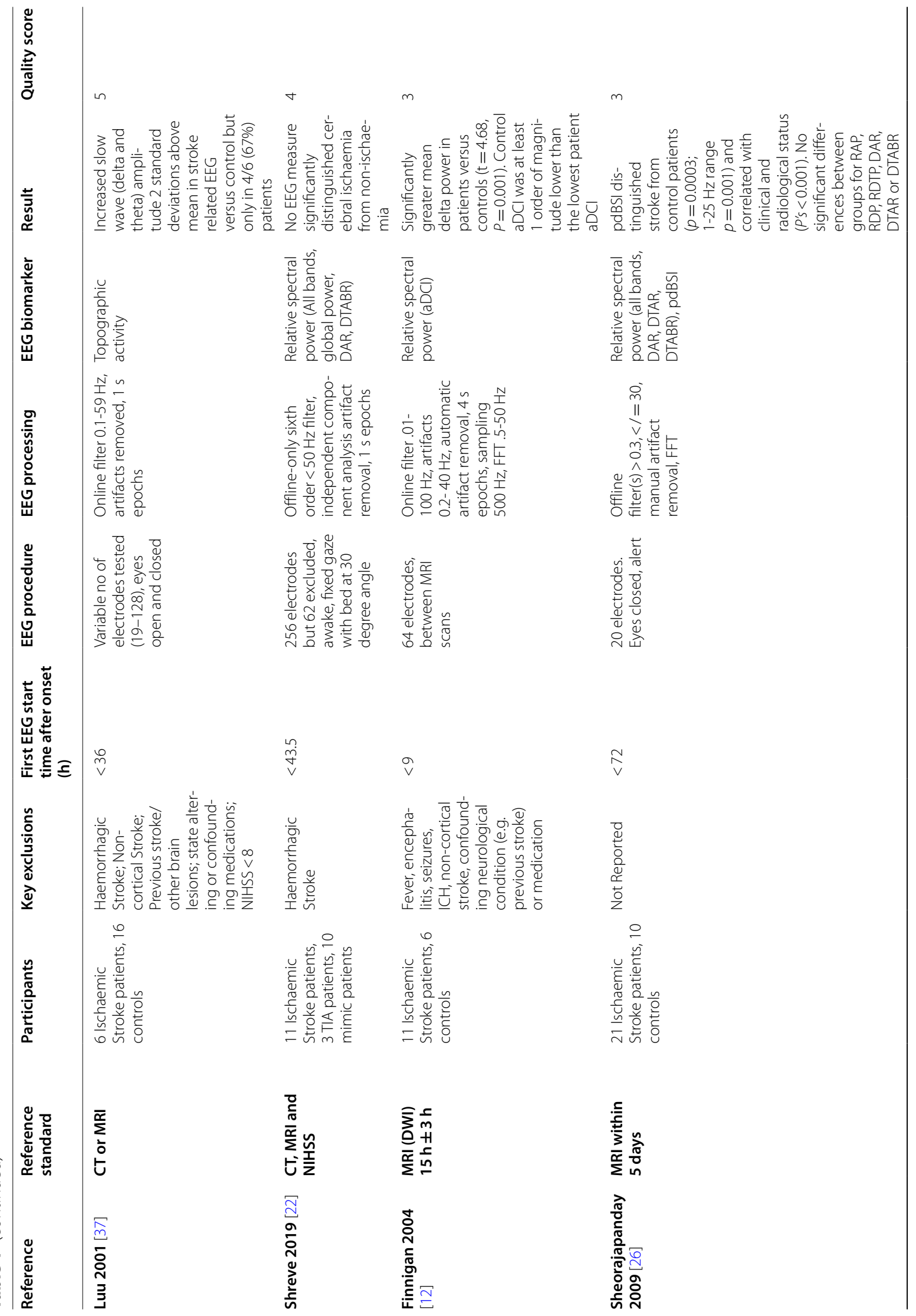




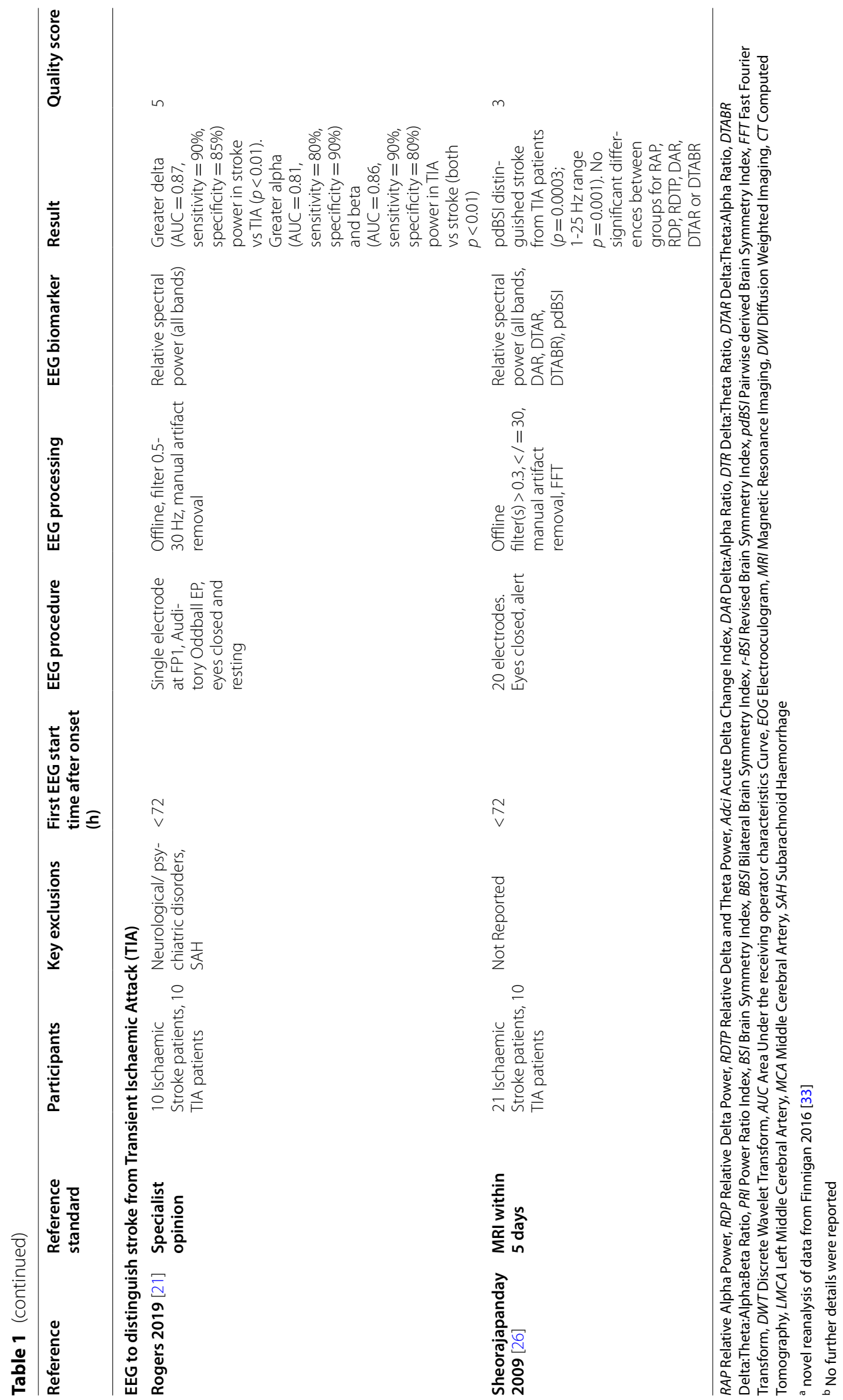


Twelve articles used absolute or relative power ratio computation as a qEEG biomarker. Greater delta power alone could differentiate between stroke and control in 11 articles [12, 21, 25, 27, 29-33, 36, 37]. Less alpha power was associated with stroke in six articles $[21,23,29,30,32,33]$. Less beta activity was associated with stroke versus non stroke in three articles [21, 29, 33]. In one article [23], stroke was associated with less high-beta $(20.5-28 \mathrm{~Hz})$ but greater low-beta frequencies $(12.5-16 \mathrm{~Hz})$. Increased theta power could identify stroke in three articles [32, 33, 37] but this was in the reverse direction for two others $[21,25]$.

Stroke was associated with a greater Delta:Alpha Ratio (DAR) in six articles [25, 29-31, 33, 35] greater Delta:Theta Ratio (DTR) in one article [25], and greater Delta:Theta:Alpha:Beta Ratio (DTABR) in three articles [30,31,33] but two articles reported that none of these EEG indices were useful [22, 26]. One article [29] showed that stroke was associated with greater Power Ratio Index (PRI) than control patients, indicating a relative increase in 'slow' (delta + theta) activity compared with 'fast' (alpha + beta) activity. Five articles used indices of brainwave symmetry between hemispheres, such as the Brain Symmetry Index (BSI) [26, 28, 30, 31, 34]. In all but one [30], greater asymmetry was shown for stroke vs control.

Amongst eight articles that calculated any summary indicator of diagnostic accuracy, performance was generally good or high [21, 23, 28, 31, 33, 35-37]. Two articles in particular displayed very high accuracy for individual EEG frequency bands but were not in complete agreement $[21,33]$. Within $24 \mathrm{~h}$ of symptom onset, Finnigan (2016) [33] reported ischaemic stroke $(n=18)$ could be detected by greater delta (AUC 0.99) and theta (AUC 0.81) activity, but less alpha (AUC 0.97) and beta (AUC 0.90) compared with control patients $(n=28)$. However, although Rogers (2019) [21] also reported accurate prediction by greater delta activity (AUC 0.87) within $72 \mathrm{~h}$ onset, there was no difference between stroke $(n=10)$ and controls $(n=10)$ for alpha and beta, and controls had greater theta activity (AUC 0.93). Finnigan (2016) [33] also reported very high AUC from higher DAR (AUC 1.0) and DTABR (AUC 0.99). Subsequent analysis confirmed that the DAR result could be replicated by using just two frontal electrodes (AUC 0.99) [35]. A more recent article used deep learning network-based modelling of clinical information and EEG data from electrode pairs selected by lasso regression within $24 \mathrm{~h}$ of symptom onset, and showed the AUC was higher (0.88) than could be achieved by standard analysis of clinical and/or EEG data [23], with slower frequencies in stroke $(n=50)$ and TIA patients $(n=13)$ versus control $(n=37)$.
For two articles also aiming to distinguish stroke from TIA, median quality score was 4 (range $3-5$ ), with small numbers of participants. One article [21] distinguished between stroke and TIA (as well as control) with high diagnostic accuracy using evoked potentials and spectral power across all bands, with greater delta, less alpha and less beta in stroke versus TIA. The other [26] distinguished stroke from TIA using a modified BSI but did not find any difference in slow:fast wave ratios.

\section{1b) Identification of ischaemic versus haemorrhagic stroke}

Only two studies considered differences between ischaemic and haemorrhagic stroke aetiologies, with differing methodologies and results. Studies are summarised in Table 2, grouped by year of publication and reference standard.

Both studies were of medium quality (median score 3.5, range 3-4). One was an examination of post-stroke seizures during EEG monitoring and found a higher incidence of these was predictive of haemorrhagic stroke [38]; extrapolated specificity was high but sensitivity low. The other used relative spectral power methods and found differences in global frequencies (i.e. a more abrupt decrease of higher frequencies in haemorrhage), but did not find any useful diagnostic value in ratios such as PRI or DAR [29].

\section{1c) Identification of ischaemic stroke due to anterior large vessel occlusion}

Five studies reported whether EEG data was associated with direct (angiographic; $n=1$ ) or indirect (infarct volume; $n=4$ ) radiological evidence that LVO was likely to be responsible for ischaemic stroke. Studies are summarised in Table 3, grouped by year of publication and reference standard.

The quality of these studies was mixed, with a median score of 3 (range 2-4). Four reported that relative spectral power detected large infarct volume (more common in LVO), either by identifying areas of increased slowerwaves (delta [12, 22] and theta [23]) and/or decreased fast-waves (beta [22, 39] and alpha [23]). Epileptiform activity (including slowing of frequencies) differentiated between territorial infarcts more typical of LVO and sub-cortical infarcts more likely to result from small vessel ischaemia [40]. Two studies comparing activity between hemispheres showed a general trend towards increased slow waves in the affected hemisphere but also a reduction in faster waves in the contralesional hemisphere when infarct size was greater [22, 40]. The only study with direct angiographic evidence of LVO [23] used deep learning models combining clinical and EEG data, showing that the combination could achieve a high level of accuracy to detect 7 cases of LVO amongst 100 


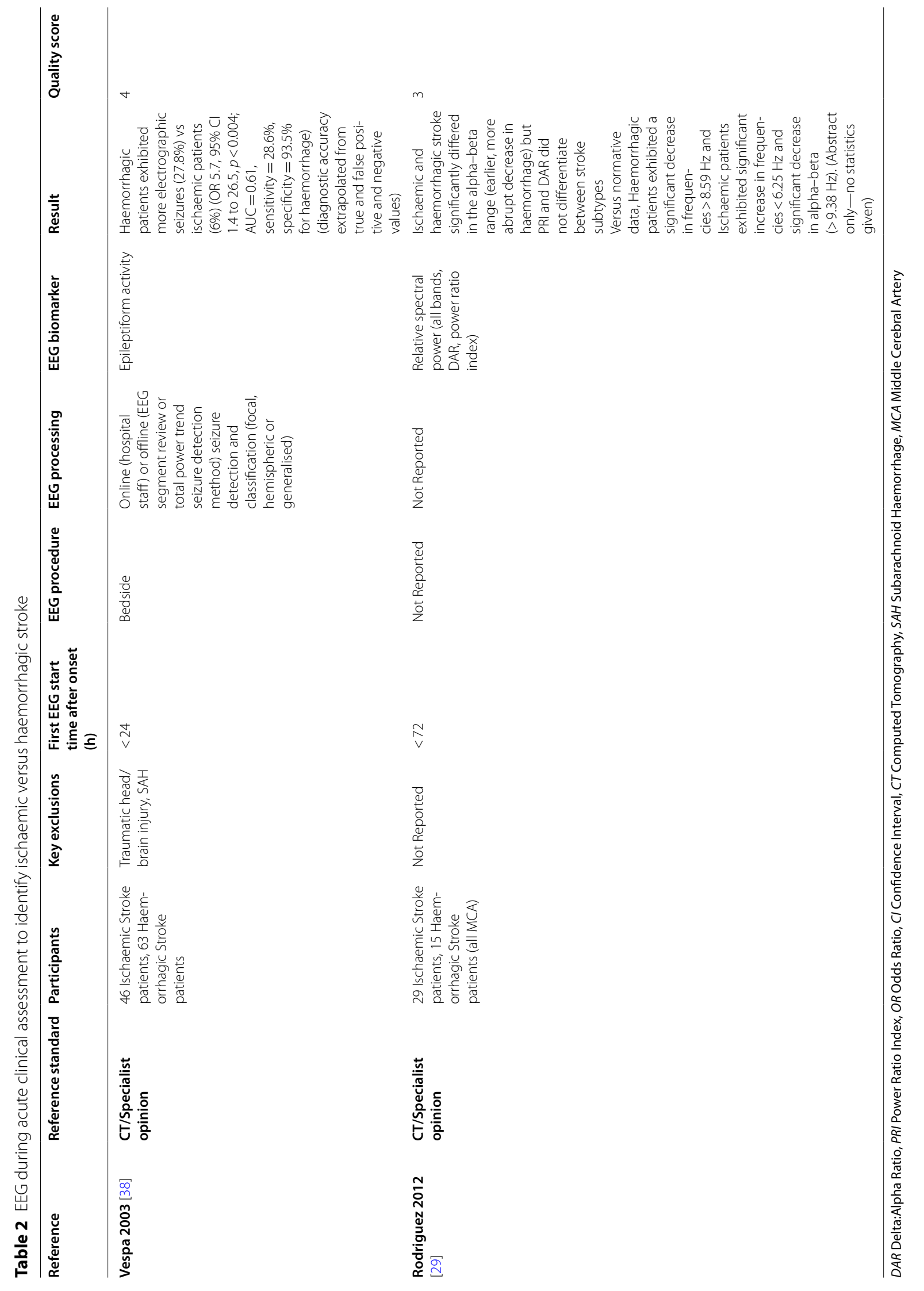




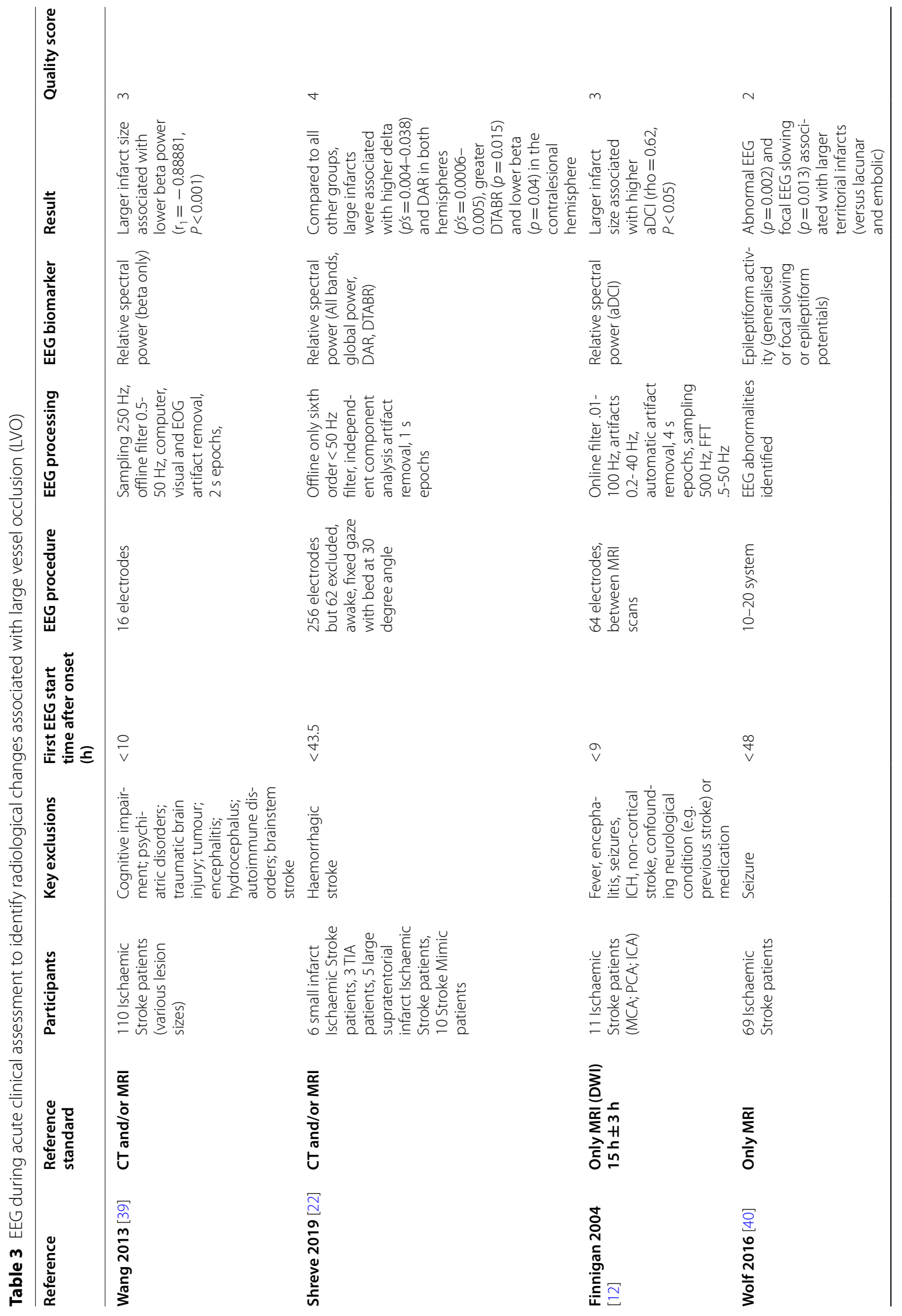




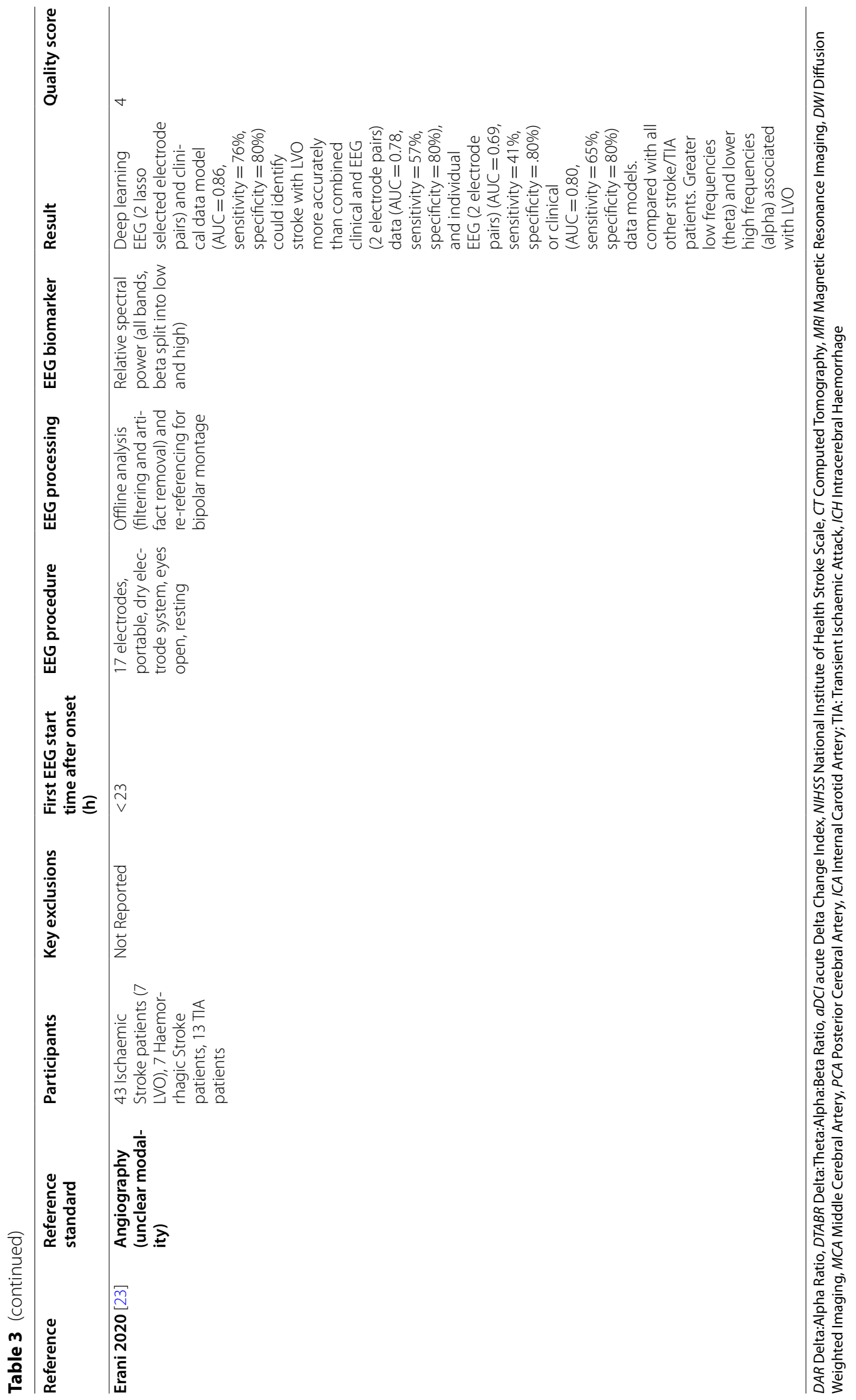


cases of suspected stroke (AUC 0.86 , sensitivity $=76 \%$, specificity $=80 \%$ ).

\section{2) Diagnostic accuracy within $6 h$ of symptom onset}

No diagnostic studies were found which consisted purely of patients within six hours of symptom onset. For identification of stroke versus non-stroke, only one small study included patients who were all within nine hours of onset [12], showing significantly greater mean delta power for stroke versus control. Studies which considered ischaemic versus haemorrhagic stroke only included patients within 24 [38] and 72 [29] hours of onset, and no conclusion can be drawn about early EEG application for this purpose. For detection of LVO, two out of five studies involved participants who were potentially within time windows for thrombectomy treatment; within nine [12] and ten [39] hours. These studies did not have high quality scores, but both showed associations with large volume infarction (loss of beta power and higher aDCI respectively) which may indicate that early changes associated with LVO are detectable.

\section{3) Prediction of outcome following confirmed stroke}

Twenty-six articles investigated the use of EEG biomarkers in predicting clinical recovery following confirmation of a stroke diagnosis within the previous $72 \mathrm{~h}$. Studies are summarised in Table 4 grouped by year of publication and outcome of interest. Prognostic articles had a median quality score of 4 , reflecting a range of scores from poor to excellent (2-5).

Twelve articles assessed outcome at various time points by dependency scales such as the modified Rankin Scale (mRS), Barthel Index/modified Barthel Index (BI/mBI), Glasgow Outcome Scale (GOS) or disability via "neurological examination". Of these, six found associations between post-stroke dependency and generalised abnormal EEG patterns such as asymmetry, slowing and epileptiform activity [41, 42, 45-47, 49]. One study found no association between EEG-recorded seizures and GOS [38]. Another eight found associations between spectral power (alpha, theta and delta) and/or spectral power ratios and poor outcome, including: more delta activity $[41,44,49,50]$, less alpha activity $[41,44,49,50]$, greater theta activity $[41,44,50,51]$ but also less theta activity [49], greater DTABR $[15,49]$ and higher Brain Symmetry Index score (denoting greater asymmetry) [48]. One study stated that qEEG frequencies predicted outcome but did not provide further detail [43]. Prognostic accuracy was variable amongst 10 articles with accuracy indices $[15,42-47,50,51,58]$, and boosted by inclusion of clinical and radiological predictors $[47,49]$.

Seven articles assessed later neurological impairment using the NIHSS. Of three studies seeking associations with abnormal EEG patterns, one found an association with epileptiform activity [38] and one generalised EEG slowing [40]. One study [54] did not report an association between epileptiform activity and the NIHSS, finding this was only useful in predicting seizure incidence. Three studies found associations between poor outcome and relative band power or ratios using various biomarkers such as less relative alpha power and greater DAR [53], greater interhemispheric alpha peak frequency asymmetry [52] and greater aDCI [12]. One study showed associations between a more favourable NIHSS after thrombolysis for ischaemic stroke and early decreases in BSI, DAR and DTABR [24]; this was the only study to focus solely on patients within six hours of symptoms onset. None of these studies calculated summary statistics to reflect accuracy.

Four studies assessed outcome by cognitive function (MoCA or diagnosis of dementia). Three found associations between spectral power and poorer cognitive outcome: lower theta, higher delta, greater DTR and DAR [25], lower beta [39], and greater theta with high background rhythm frequency [56]. One older study simply associated "abnormal EEG", such as abnormal foci and background slowing, with risk of developing dementia [55]. Prognostic accuracy was low for any 'abnormal EEG' recording [55] but high (sensitivity 92.3\%) if low beta activity was present [39].

Four studies considered prediction of mortality at various time intervals after stroke. At hospital discharge, greater contralateral theta power [57] and greater asymmetry measured by the Bilateral Brain Symmetry Index (BBSI) [28] were associated with poorer outcome. Higher DAR at day 90 [30], greater contralateral theta power at 6 months [57], and epileptiform activity, background slowing and overall asymmetry at 12 months [47] were associated with poorer outcome. Prognostic accuracy was moderate, but with poor sensitivity, in two studies $[47,57]$ and appeared high for two other studies $[28,30]$.

\section{Discussion}

In this review, we have summarised published literature on the use of EEG in the diagnosis and prognosis of stroke when applied within $72 \mathrm{~h}$ of onset. Due to variability in study design and EEG technology, we did not plan to directly compare clinical utility of EEG biomarkers or perform a meta-analysis. Despite limitations in study quality, such as unclear inclusion criteria and reference standards, reports generally support further development and evaluation of EEG techniques to examine their ability to facilitate accurate clinical stratification of patients with stroke symptoms.

There is evidence to support potentially valuable diagnostic accuracy of EEG approaches for differentiating 


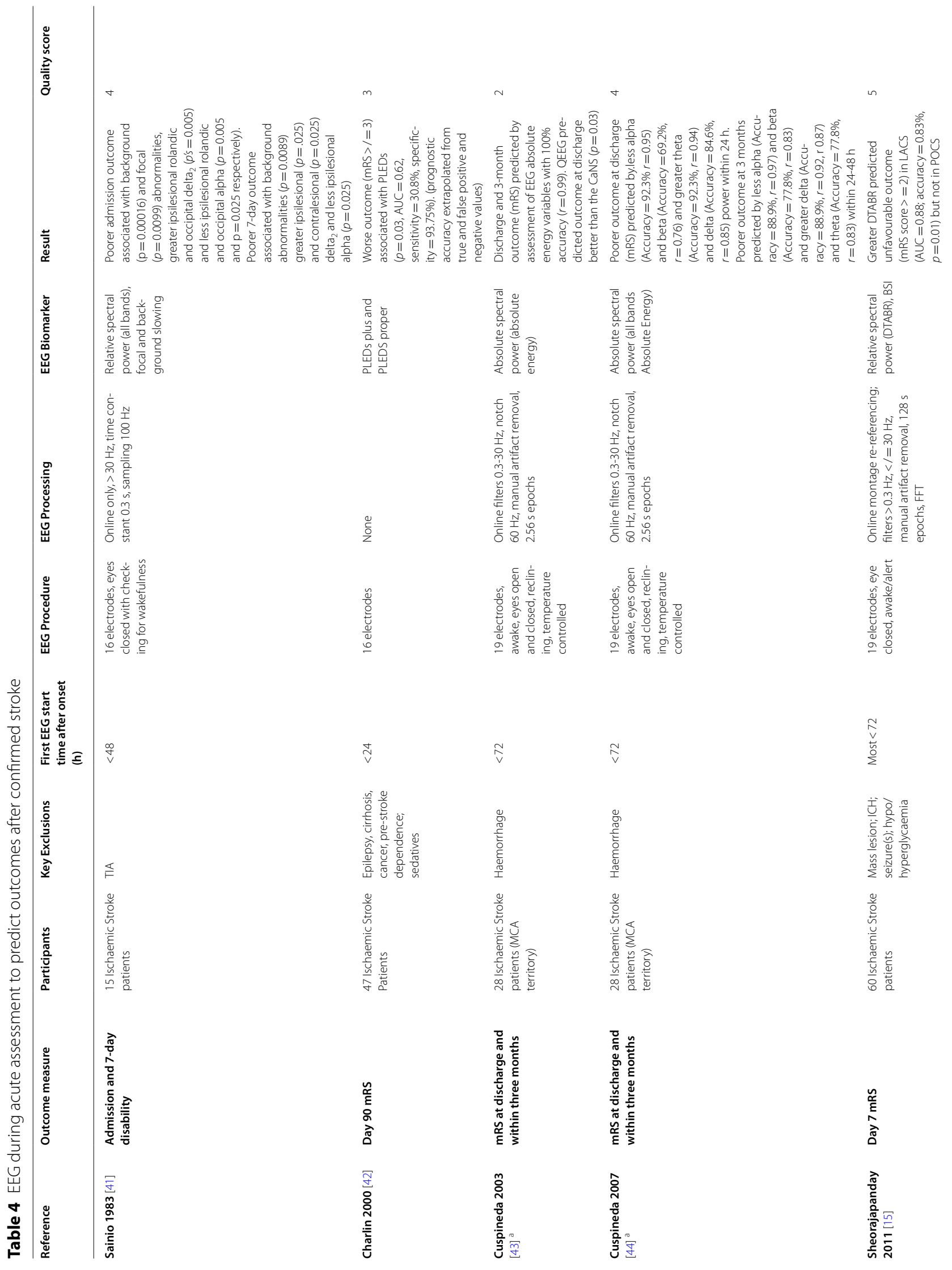




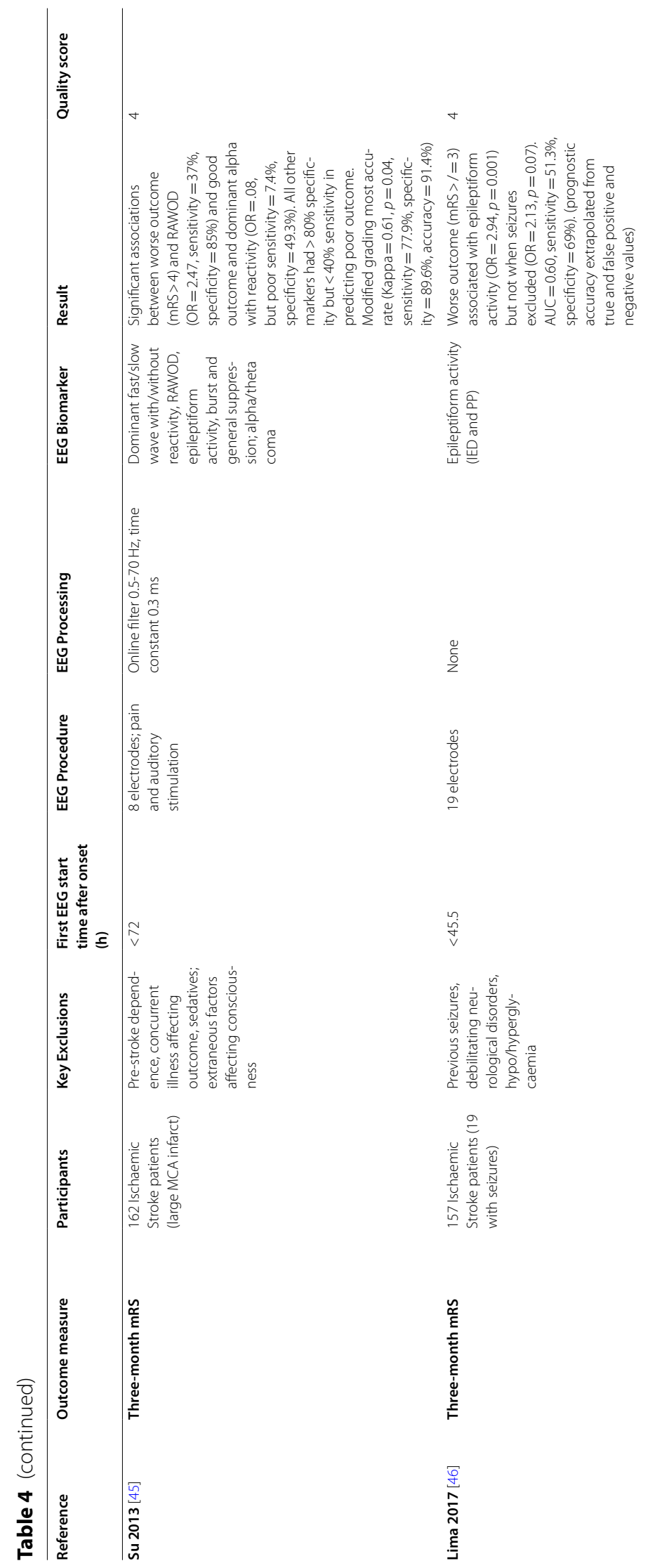




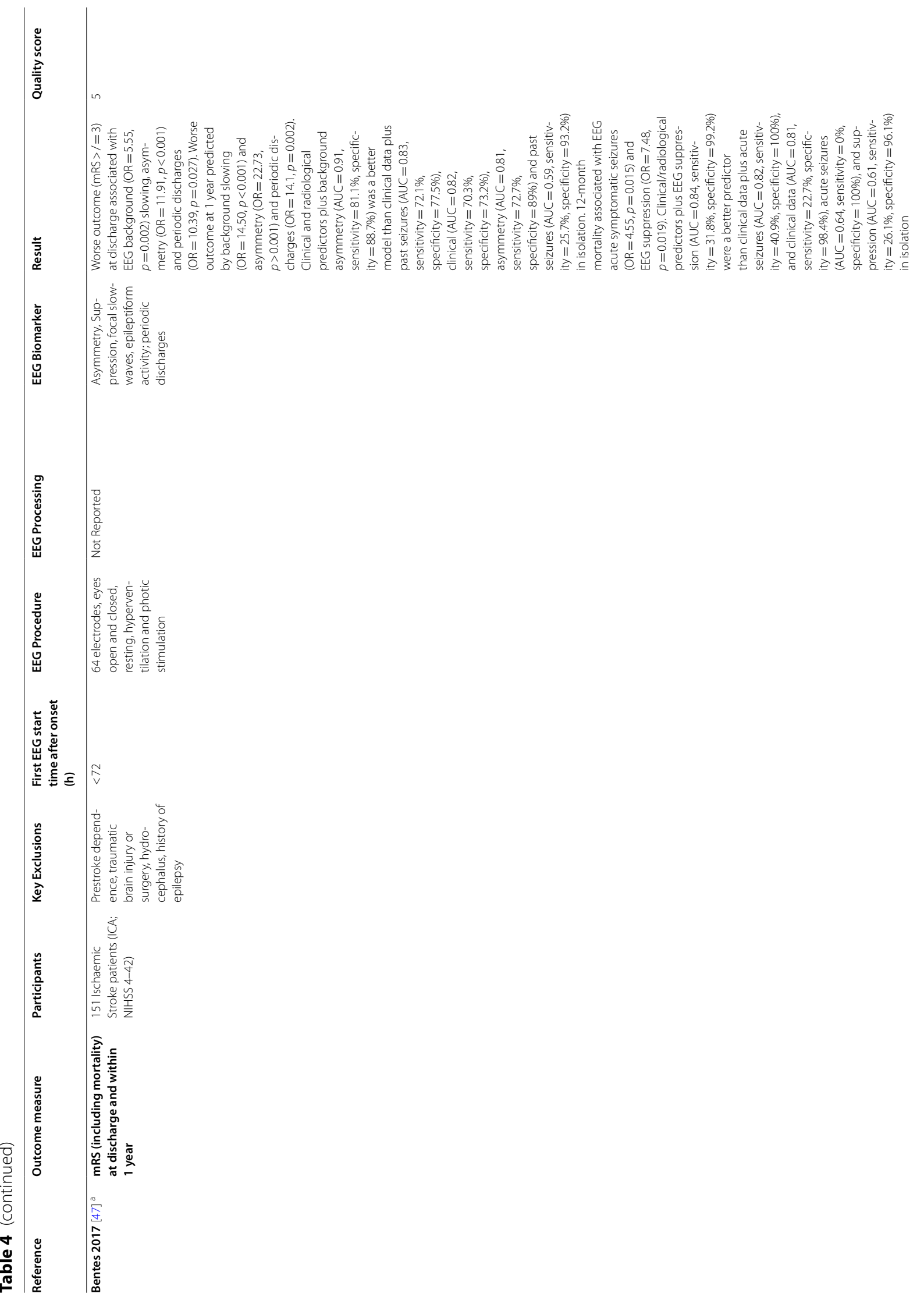




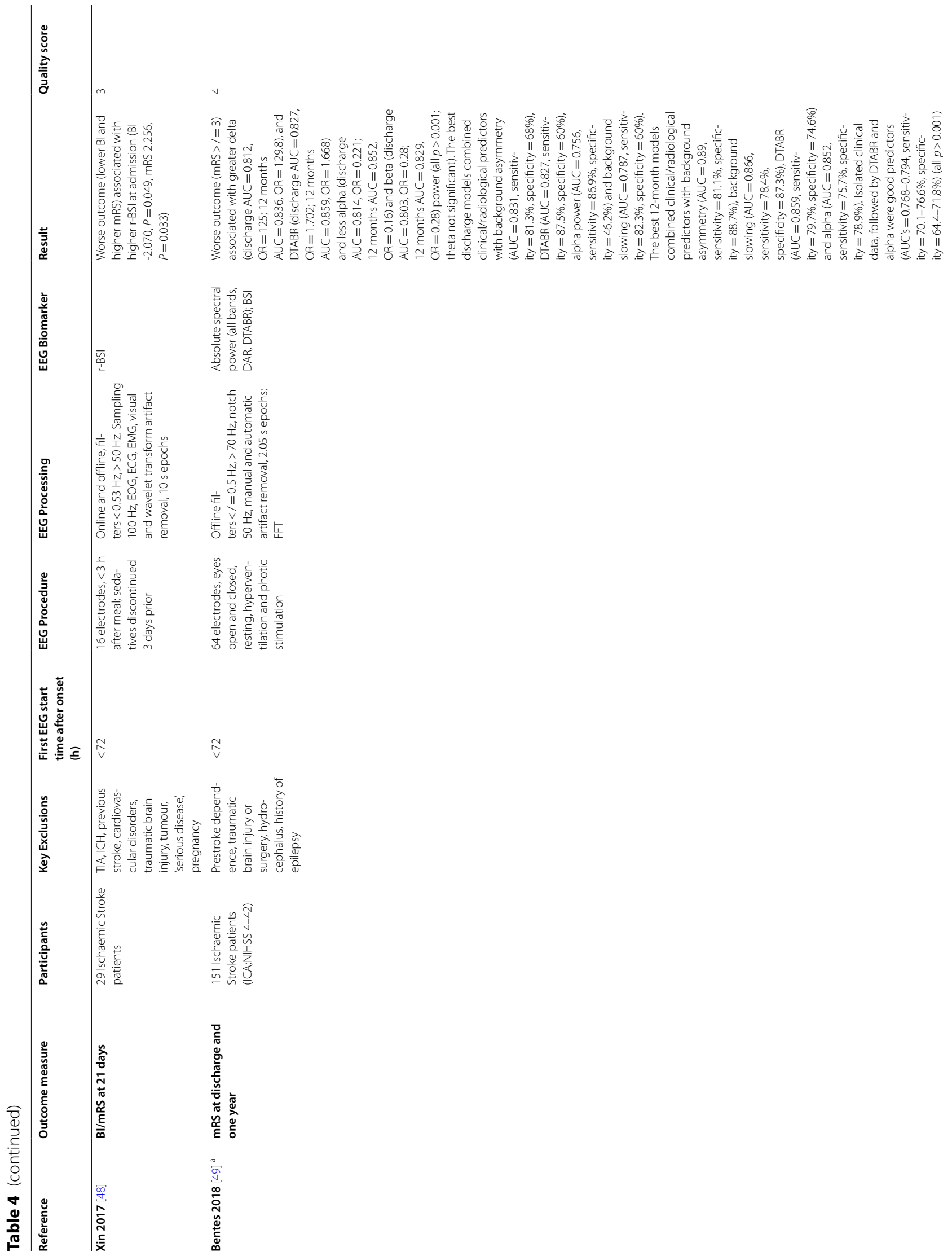




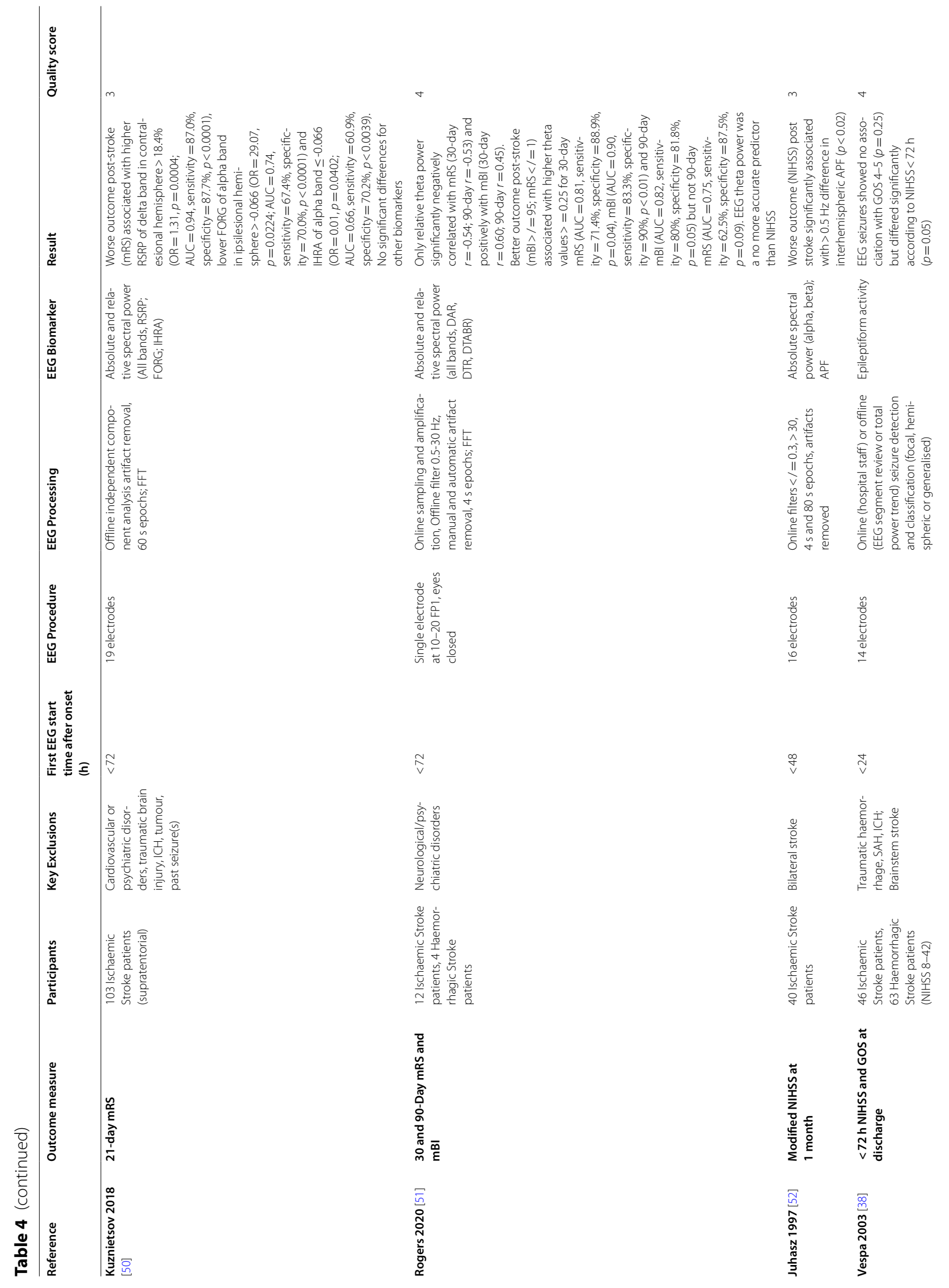




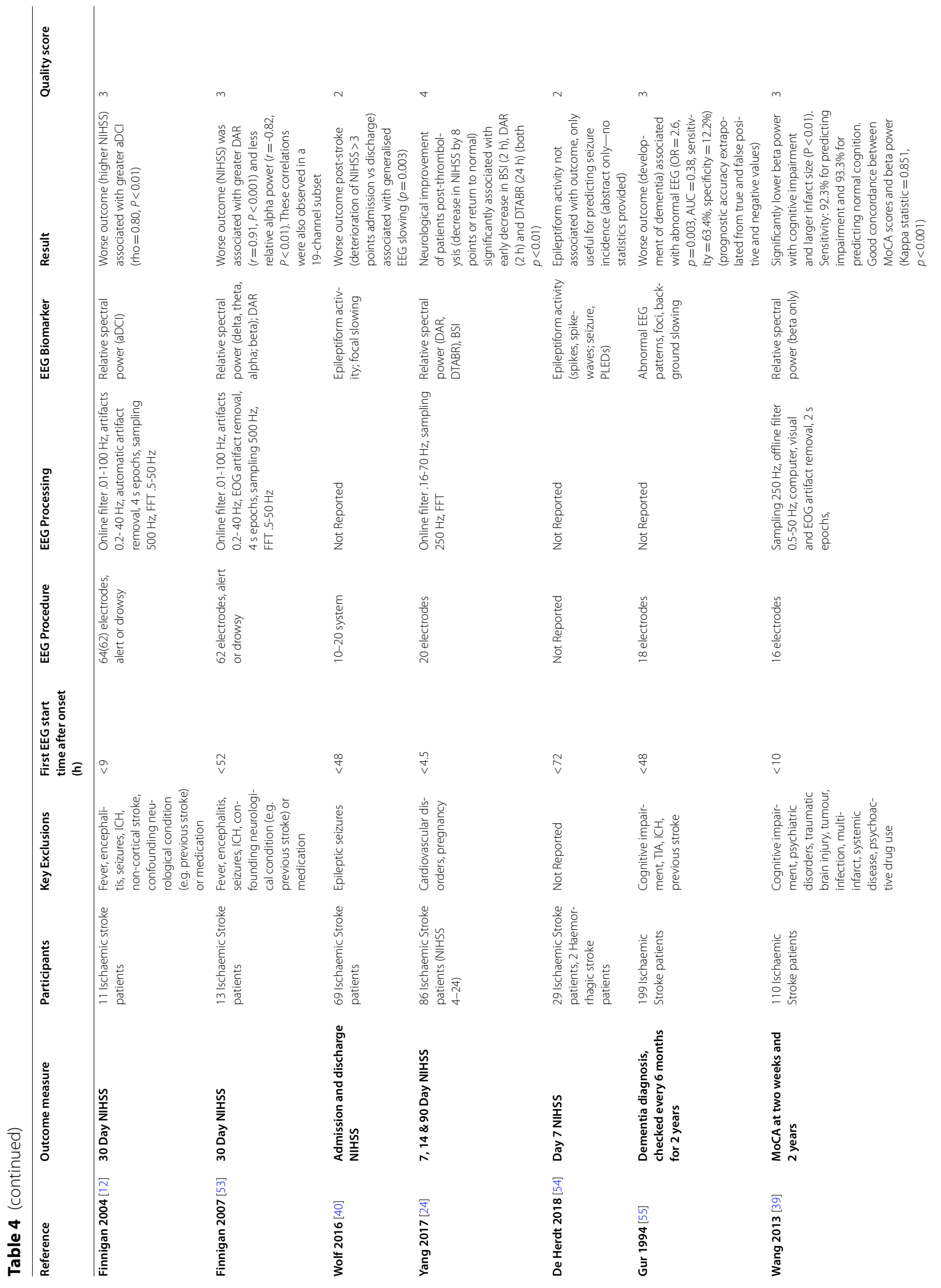




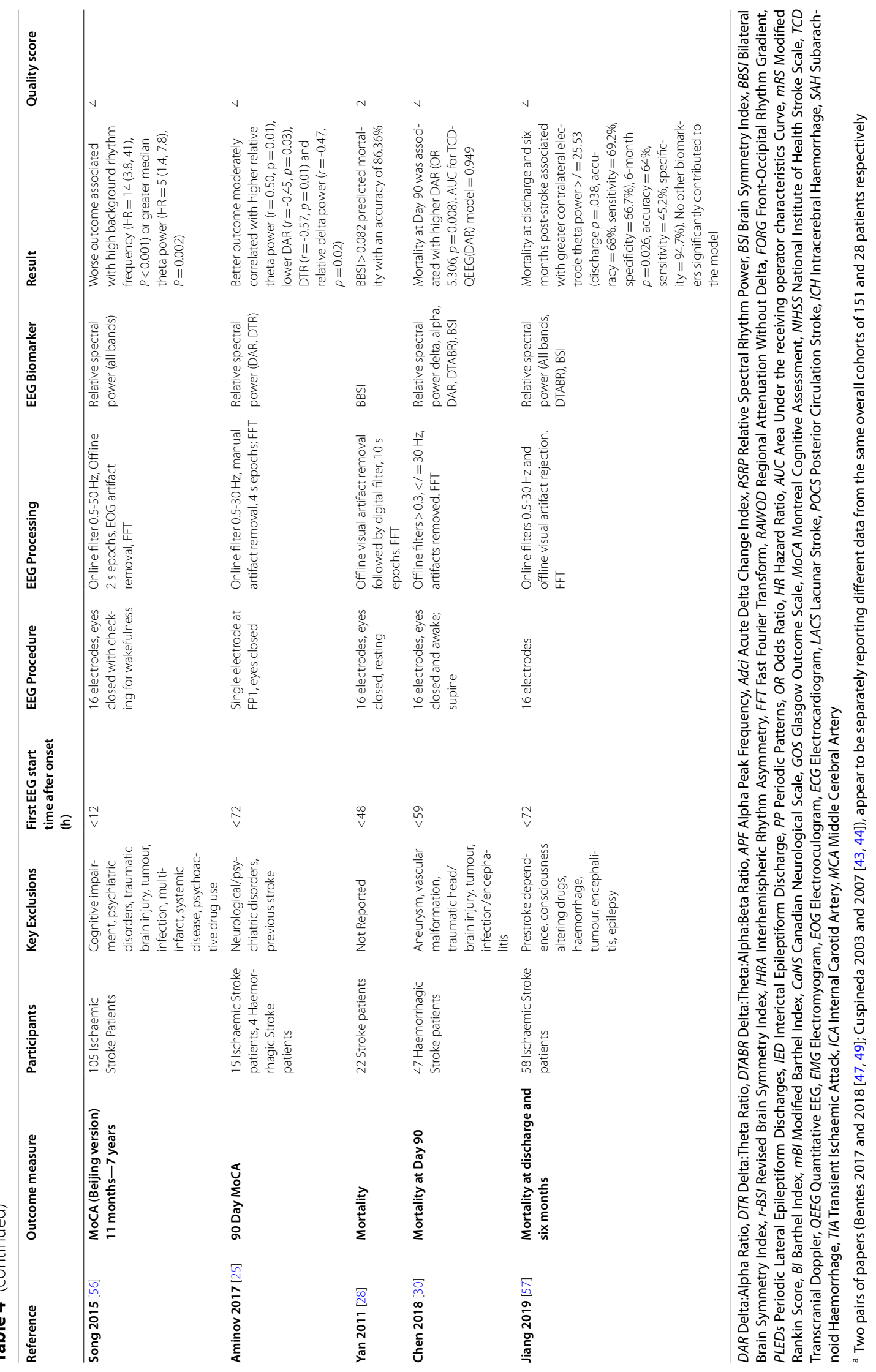


stroke from non-stroke states due to statistical associations between a diagnosis of stroke, increased slowwave EEG activity (delta in particular) and decreased fast-wave activity (alpha and beta). Although theta activity was often increased for stroke relative to control subjects, this was not a consistent finding and it appears to be the least useful frequency for diagnosis in this context, probably due to its intermediate speed between alpha (fast) and delta (slow). Two studies found greater asymmetry and slow-wave activity in stroke versus TIA, but it should be noted that both recruited patients within $72 \mathrm{~h}$ of symptom onset, during which TIA symptoms would have resolved, and so the results would have little value in discriminating stroke versus TIA or in assisting with therapeutic decision making. However, EEG may have value in differentiating TIA and stroke mimic patients which could have implications for future secondary prevention. Despite these promising early studies, it is important to recognise that most were small and included selected patients who were beyond six hours since symptom onset, so there is relatively little evidence that the potential EEG biomarkers identified would be present in the very early stages of stroke when the impact for emergency care decisions would be greatest e.g. to initiate direct ambulance transfer to a stroke centre rather than a general hospital without specialist care.

Evaluation of the ability of EEG to distinguish between haemorrhagic and ischaemic stroke was limited to two studies, which again were not focussed upon the early hours when this information would be of greatest clinical value e.g. for administration of thrombolytic therapy within $4.5 \mathrm{~h}$ of symptom onset [38]. Because stroke due to haemorrhage typically exhibits greater symptom severity at presentation [59] and as neither study adjusted for patient characteristics, it is also unknown whether the observed EEG differences are attributable to the underlying stroke type. Currently it appears unlikely that EEG has a role to play in the differentiation between ischaemic and haemorrhagic stroke that would change patient management.

On the basis that indirect radiological evidence is a reliable indicator of LVO, a small number of studies support the further development of EEG biomarkers for this purpose, although only two studies focused on a suspected stroke population during the standard time interval of maximal clinical value for thrombectomy [12, 39]. For all studies, it was unclear whether participants were fully representative of an unselected suspected stroke population, and it remains uncertain whether it will be possible to accurately transfer suitable patients directly to thrombectomy centres. Only one study used angiography as the reference standard and reported that the most promising AUC (0.86) was achieved by combining clinical and EEG (lower alpha and greater theta) data with a deep learning algorithm [23]. In addition, a prospective study published since completion of our search has also confirmed that amongst 109 patients within $24 \mathrm{~h}$ of symptom onset (25 angiography-proven LVO, 38 nonLVO ischemic, 14 haemorrhages, and 32 stroke mimics) an AUC of 0.88 was achieved using a portable LVOdetection device which combined EEG and somatosensory-evoked potentials [60].

When used to provide an early estimate of prognosis, EEG biomarkers recorded within $72 \mathrm{~h}$ of stroke onset had associations with later clinical outcomes which could be useful to inform acute management decisions including future dependency, neurological impairment, cognitive function and mortality. In particular, greater delta and theta activity, less alpha and beta activity, greater interhemispheric asymmetry and greater DAR and DTABR ratios appear to be moderate predictors for both long-, and short-term neurological function and dependency. Such associations are not unexpected as EEG changes reflect the volume of cerebral tissue injury, which itself directly correlates with dependency and survival [61]. Only one cohort was identified where EEG information improved upon the accuracy of predictions for dependency and mortality made using simple clinical assessments and/or brain imaging to confirm the number and location of vascular lesions [47, 49]. There are, however, validated clinical scores already available to estimate various aspects of physical stroke recovery (e.g. arm function [62]; independent walking ability [63]) which are not widely used in practice because of concerns that they could restrict access to finite, but potentially beneficial, care resources [64]. Therefore, in parallel with further research focussed upon whether surface EEG can refine early clinical prediction of future survival and dependency, it is necessary to understand whether using such technology as a decision support aid is an acceptable concept and how the results would be communicated to patients and their families. Likewise, although clinical models to predict cognitive impairment after stroke have been created, currently there is insufficient evidence of validity and/or accuracy for routine use [65]. Neuroimaging variables such as white matter lesions have separately been found to be risk factors for dementia after stroke [66] and so it is feasible that EEG biomarkers will be helpful in identifying patients with a subclinical risk. However, studies identified by our search did not combine EEG data with neuroimaging variables, or compare to age matched controls, and it will be necessary to undertake additional longitudinal studies of well described cohorts before it is clearer whether EEG has a role to play as a clinical decision aid 
by providing prognostic estimates during acute stroke care.

According to the basic scoring system we employed, most studies were not high quality, usually due to a lack of clarity about populations, reference standards and adjudication. Few studies produced power calculations or seemed to be adequately powered given required case:variable ratios. Many studies did not calculate prognostic or diagnostic accuracy or provide information that would be important to determine clinical utility, such as the number of patients who could not tolerate the procedure and the time required to obtain an EEG recording. Techniques using large numbers of electrodes are unlikely to be deployed during emergency assessment of suspected stroke if application requires additional training and significantly delays routine care, but it is encouraging that diagnostic value was reported by studies using six electrodes or fewer [21, 34, 35]. Clinical feasibility will be further facilitated by easily applicable dry (sans electroconductive gel) electrode systems, and ongoing development of machine learning approaches to automatically select electrode pairs and rapidly identify multi-wave activity patterns predictive of a stroke diagnosis or LVO [23, 60]. Rapid application is less essential for collecting information to inform prognosis and could be done after hospital arrival, but it is still important to consider that some patients may not be able to tolerate a lengthy EEG procedure and efficient portable systems will minimise disruption of acute clinical care.

As well as high accuracy and rapid electrode application, other characteristics have been described that would make diagnostic technology more suitable for prehospital use including: a user-friendly interface with an easily interpretable output that enables operation by ambulance practitioners with relatively low exposure to stroke, a rugged compact design enabling storage in ambulances, and a cost which is affordable relative to the impact upon patient care and outcomes [67]. For prehospital EEG deployment, it would also be important that ambulance practitioners receive clear instruction about which suspected stroke patients should have a reading taken. For instance, Bayesian modelling has shown that because ischaemic stroke patients with low NIHSS scores are unlikely to have LVO whilst those with high scores are very likely to have LVO, a diagnostic would have greatest population level impact when used for patients with intermediate symptom severity e.g. (Additional file 1) NIHSS scores of 5 to 10 points [68]. Other considerations influencing the utility of diagnostic technology during prehospital assessment are how often patients develop acute complications (including seizures), the prevalence of pre-existing co-morbidities that limit emergency treatment options, and the location of the incident relative to the nearest stroke centre.

\section{Limitations}

Finally, our review has some limitations which should be acknowledged. It was not possible to include studies written in a non-English language, which may have excluded relevant reports that did not already have an English translation available. There was a wide variation in EEG technique (e.g. filters and electrode placement) and outcome measures which prevented data meta-analysis and hinders recommendation of a specific technical approach for diagnosis or prognosis. Additionally, these cohorts were specifically selected for research and most studies had strict inclusion criteria to minimise interference with the EEG signal, limiting the generalisability of findings to the wider suspected stroke population, especially in the prehospital setting. In many studies it was unclear which diagnostic reference standards were used and even when these were described, a lack of standardisation limited interpretation of the results. Lastly, as none of these studies were conducted in a prehospital setting, the findings cannot be directly extrapolated to the more challenging ambulance environment, where useful diagnostic technology should be able to detect very early changes of brain tissue amongst a heterogeneous population.

\section{Future directions}

There have been recent advances in commercial EEG technology for use in stroke diagnosis, notably for early identification of LVO [18], but our review was limited to published studies. Based on results to date, it is important to continue to evaluate these devices in real world populations to determine which combination of EEG data might assist with early triage of suspected stroke. An ideal diagnostic accuracy study design would prospectively collect blinded EEG readings during prehospital clinical assessment and compare the performance of a predetermined data algorithm to an independently adjudicated reference standard which is appropriate for the intended purpose e.g. in a study to identify LVO, the reference standard would require all ischaemic stroke participants to undergo angiography to confirm or exclude occlusions in pre-specified locations of the cerebral vasculature. The sample size should be justified in advance by a calculation describing a clinically meaningful minimum level of performance, and the participant inclusion criteria should reflect a relevant clinical population e.g. if the purpose is to facilitate thrombolysis, a maximum of $4.5 \mathrm{~h}$ should be allowed between symptom onset and EEG reading. Important operational information must be reported to assess feasibility such as technical failures and the time taken to 
apply electrodes. If satisfactory EEG diagnostic accuracy is demonstrated and the technology appears to be acceptable for clinical deployment, further trials should evaluate the real-world impact upon the efficiency of stroke care pathways, including its overall cost-effectiveness during delivery of emergency therapies. Other portable technologies are also in development for emergency detection of stroke and LVO, including blood assays and non-ionising imaging [8], and the future clinical value of surface EEG should be considered alongside alternative biomarkers used separately and in combination.

\section{Conclusion}

Reports identified during this review show that surface EEG techniques have promise for assisting with stroke diagnosis and prognosis during the acute phase. However due to the small size of studies and variations in technology, populations and settings, it is not yet possible to make recommendations regarding EEG use to guide early diagnostic and prognostic management decisions. Further research is required to determine which combinations of electrodes, waveforms, clinical data and neuroimaging variables can accurately stratify unselected populations into clinically important subgroups, and to confirm that EEG application for this purpose is both acceptable and feasible within the first few hours after symptom onset.

\begin{abstract}
Abbreviations
aDCl: Acute Delta Change Index; APF: Alpha Peak Frequency; AUC: Area Under the receiving operator characteristics Curve; BI: Barthel Index; BSI: Brain Symmetry Index; BBSI: Bilateral Brain Symmetry Index; CaNS: Canadian Neurological Scale; Cl: Confidence Interval; CT/CTA: Computed Tomography/Computed Tomography Angiography; DAR: Delta:Alpha Ratio; DTABR: Delta:Theta:Alpha:Beta Ratio; DTAR: Delta:Theta:Alpha Ratio; DTR: Delta:Theta Ratio; DWT: Discrete Wavelet Transform; DWI: Diffusion Weighted Imaging; ECG: Electrocardiogram; ED: Emergency Department; EEG: Electroencephalogram; EMG: Electromyogram; EOG: Electrooculogram; FFT: Fast Fourier Transform; FORG: Fronto-Occipital Rhythm Gradient; GOS: Glasgow Outcome Scale; HR: Hazard Ratio; ICA: Internal Carotid Artery; ICH: Intracerebral Haemorrhage; IED: Interictal Epileptiform Discharge; IHRA: Interhemispheric Rhythm Asymmetry; LACS: Lacunar Stroke; LMCA: Left Middle Cerebral Artery; LVO: Large Vessel Occlusion; mBl: Modified Barthel Index; MCA: Middle Cerebral Artery; MoCA: Montreal Cognitive Assessment; MRI/A: Magnetic Resonance Imaging/ Angiography; mRS: Modified Rankin Scale; NIHSS: National Institutes of Health Stroke Scale; OR: Odds Ratio; PCA: Posterior Cerebral Artery; pdBSI: Pairwise derived Brain Symmetry Index; PLEDs: Periodic Lateral Epileptiform Discharges; POCS: Posterior Circulation Stroke; PP: Periodic Patterns; PRI: Power Ratio Index; PRISMA: Preferred Reporting Systems for Systematic Reviews and MetaAnalyses; RAP: Relative Alpha Power; RAWOD: Regional Attenuation Without Delta; RDP: Relative Delta Power; RDTP: Relative Delta and Theta Power; RSRP: Relative Spectral Rhythm Power; r-BSI: Revised Brain Symmetry Index; SAH: Subarachnoid Haemorrhage; TIA: Transient Ischaemic Attack; TCD:Transcranial Doppler ultrasonography; QEEG: Quantitative EEG.
\end{abstract}

\section{Supplementary Information}

The online version contains supplementary material available at https://doi. org/10.1186/s12873-022-00585-w.

Additional file 1.

\section{Acknowledgements}

We are grateful to Alex Inskip (Newcastle University) for assistance with designing the search strategy.

\section{Authors' contributions}

CP and LSh conceived the review. CP, LSh, LSu and RF developed the review methods. LSu and CP designed and conducted the search strategy. LSu and RF, with assistance of CP and LSh, assessed studies for inclusion. LSu, HL and RF extracted data from included studies. $\mathrm{LSu}, \mathrm{HL}, \mathrm{CP}$ and RF drafted the manuscript. LSu, HL, CP, LSh and RF were involved in the interpretation of data, critically reviewed the manuscript for intellectual content and approved the final version of the manuscript. LS and HL contributed equally and are joint first authors.

\section{Funding}

$\mathrm{CP}$ and LSu received salary funding from the UK Stroke Association during production of the review.

\section{Availability of data and materials}

Data sharing is not applicable to this article as no datasets were generated or analysed during the current study.

\section{Declarations}

Ethics approval and consent to participate

Not applicable.

\section{Consent for publication}

Not applicable.

\section{Competing interests}

$\mathrm{HL}$, LSu and RF declare no conflicts of interest. CP and LSh declare interests as investigators for two non-commercial studies of new technologies to assist with stroke assessment (PRISM: http://www.isrctn.com/ISRCTN22323981 and ABACUS: http://www.isrctn.com/ISRCTN79169844).

Received: 20 April 2021 Accepted: 4 February 2022

Published online: 28 February 2022

\section{References}

1. Feigin VL, Forouzanfar MH, Krishnamurthi R, Mensah GA, Connor M, Bennett DA, et al. Global and regional burden of stroke during 1990-2010: findings from the Global Burden of Disease Study 2010. Lancet. 2014;383(9913):245-54.

2. National Audit Office. Reducing brain damage: faster access to better stroke care. Health and Social Care. London 2005. https://www.nao.org. uk/report/department-of-health-reducing-brain-damage-faster-accessto-better-stroke-care/

3. Clinical Effectiveness and Evaluation Unit Royal College of Physicians. The Sentinel Stroke National Audit Programme (SSNAP). 2020. https://www. rcplondon.ac.uk/projects/ssnap-clinical-audit.

4. McClelland G, Rodgers $\mathrm{H}$, Flynn D, Price Cl. The frequency, characteristics and aetiology of stroke mimic presentations: a narrative review. Eur J Emerg Med. 2019;26(1):2-8.

5. Turc G, Maier B, Naggara O, Seners $P$, Isabel $C$, Tisserand $M$, et al. Clinical Scales Do Not Reliably Identify Acute Ischemic Stroke Patients With Large-Artery Occlusion. Stroke. 2016;47(6):1466-72.

6. Smith EE, Kent DM, Bulsara KR, Leung LY, Lichtman JH, Reeves MJ, et al. Accuracy of Prediction Instruments for Diagnosing Large Vessel Occlusion in Individuals With Suspected Stroke: A Systematic Review for the 2018 Guidelines for the Early Management of Patients With Acute Ischemic Stroke. Stroke. 2018;49(3):e111-22.

7. Fassbender K, Merzou F, Lesmeister M, Walter S, Grunwald IQ, RagoschkeSchumm A, Bertsch T, Grotta J. Impact of mobile stroke units. J Neurol Neurosurg Psychiatry. 2021;92(8):815-22.

8. Lumley HA, Flynn D, Shaw L, McClelland G, Ford GA, White PM, Price Cl. A scoping review of pre-hospital technology to assist ambulance personnel 
with patient diagnosis or stratification during the emergency assessment of suspected stroke. BMC Emerg Med. 2020;20(1):1-21.

9. Tolonen U, Sulg IA. Comparison of quantitative EEG parameters from four different analysis techniques in evaluation of relationships between EEG and CBF in brain infarction. Electroencephalogr Clin Neurophysiol. 1981;51(2):177-85.

10. Jordan KG. Emergency EEG and continuous EEG monitoring in acute ischemic stroke. J Clin Neurophysiol. 2004;21(5):341-52.

11. Finnigan $S$, van Putten MJ. EEG in ischaemic stroke: quantitative EEG can uniquely inform (sub-)acute prognoses and clinical management. Clin Neurophysiol. 2013;124(1):10-9.

12. Finnigan $S P$, Rose $S E$, Walsh M, Griffin M, Janke AL, McMahon KL, et al. Correlation of quantitative EEG in acute ischemic stroke with 30-day NIHSS score: comparison with diffusion and perfusion MRI. Stroke. 2004;35(4):899-903.

13. Sheorajpanday RV, Nagels G, Weeren AJ, van PuttenDeyn MJPPDe. Quantitative EEG in ischemic stroke: correlation with functional status after 6 months. Clin Neurophysiol. 2011;122(5):874-83.

14. Finnigan SP, Rose SE, Chalk JB. Rapid EEG changes indicate reperfusion after tissue plasminogen activator injection in acute ischaemic stroke. Clin Neurophysiol. 2006;117(10):2338-9.

15. Sheorajpanday RV, Nagels G, Weeren AJ, De Deyn PP. Quantitative EEG in ischemic stroke: correlation with infarct volume and functional status in posterior circulation and lacunar syndromes. Clin Neurophysiol. 2011;122(5):884-90.

16. van Putten MJ, Tavy DL. Continuous quantitative EEG monitoring in hemispheric stroke patients using the brain symmetry index. Stroke. 2004;35(11):2489-92.

17. Jakab A, Kulkas A, Salpavaara T, Kauppinen P, Verho J, Heikkila H, et al. Novel wireless electroencephalography system with a minimal preparation time for use in emergencies and prehospital care. Biomed Eng Online. 2014;13:60.

18. Walsh KB. Non-invasive sensor technology for prehospital stroke diagnosis: Current status and future directions. Int J Stroke. 2019;14(6):592-602.

19. Cloostermans MC, de Vos CC, van Putten MJ. A novel approach for computer assisted EEG monitoring in the adult ICU. Clin Neurophysiol. 2011;122(10):2100-9.

20. Tricco AC, Lillie E, Zarin W, O'Brien KK, Colquhoun H, Levac D, et al. PRISMA Extension for Scoping Reviews (PRISMA-SCR): Checklist and Explanation. Ann Int Med. 2018;169(7):467-73.

21. Rogers JM, Bechara J, Middleton S, Johnstone SJ. Acute EEG Patterns Associated With Transient Ischemic Attack. Clin EEG Neurosc. 2019;50(3):196-204.

22. Shreve L, Kaur A, Vo C, Wu J, Cassidy JM, Nguyen A, et al. Electroencephalography Measures are Useful for Identifying Large Acute Ischemic Stroke in the Emergency Department. J Stroke Cerebrovasc Dis. 2019;28(8):2280-6.

23. Erani F, Zolotova N, Vanderschelden B, Khoshab N, Sarian H, Nazarzai L, et al. Electroencephalography Might Improve Diagnosis of Acute Stroke and Large Vessel Occlusion. Stroke. 2020;51(11):3361-5.

24. Yang JZ, Xiaohui. Bai, Qingke. Zhao, Zhengguo. Sui, Haijing. Quantitative electroencephalograph in acute ischemic stroke treated with intravenous recombinant tissue plasminogen activator. Int J Clin Exp Pathol. 2017;10:507-14.

25. Aminov A, Rogers JM, Johnstone SJ, Middleton S, Wilson PH. Acute single channel EEG predictors of cognitive function after stroke. PLoS One. 2017;12(10):e0185841.

26. Sheorajpanday RV, Nagels G, Weeren AJ, van Putten MJ, De Deyn PP. Reproducibility and clinical relevance of quantitative EEG parameters in cerebral ischemia: a basic approach. Clinical Neurophysiology. 2009;120(5):845-55.

27. Cohen BA, Bravo-Fernandez EJ, Sances A Jr. Automated electroencephalographic analysis as a prognostic indicator in stroke. Med Biol Eng Comput. 1977;15(4):431-7.

28. Yan M, Hou Z, Gao Y. A bilateral brain symmetry index for analysis of EEG signal in stroke patients. 4th International Conference on Biomedical Engineering and Informatics (BME), Shanghai. 2011:pp. 8-11.

29. Rodriguez VH, FMontoya A, Bosch J. Value of qEEG parameters for the early differentiation between ischemic and hemorrhagic stroke. Journal of Clinical Neurophysiology. 2012;29(6):528.
30. Chen Y, Xu W, Wang L, Yin X, Cao J, Deng F, et al. Transcranial Doppler combined with quantitative EEG brain function monitoring and outcome prediction in patients with severe acute intracerebral hemorrhage. Critical Care. 2018;22(1):36.

31. Chan JYW, S. K. Prawito, P. Rahma, O. N. ELM (Extreme Learning Machine) Method for Detecting Acute Ischemic Stroke using Conventional and Specific Asymmetry BSI (Brain Symmetry Index) features based on EEG Signals. AIP Conf Proc. 2019;2092:2092

32. Machado C, Cuspineda E, Valdes P, Virues T, Llopis F, Bosch J, et al. Assessing acute middle cerebral artery ischemic stroke by quantitative electric tomography. Clinical EEG Neuroscience. 2004;35(3):116-24.

33. Finnigan $\mathrm{S}$, Wong $\mathrm{A}$, Read $\mathrm{S}$. Defining abnormal slow EEG activity in acute ischaemic stroke: Delta/alpha ratio as an optimal QEEG index. Clin Neurophysiol. 2016;127(2):1452-9.

34. Gottlibe M, Rosen O, Weller B, Mahagney A, Omar N, Khuri A, et al. Stroke identification using a portable EEG device - A pilot study. Clin Neurophysiol. 2020;50(1):21-5.

35. Finnigan S, Wong A. Towards pre-hospital identification of acute ischemic stroke: The value of QEEG from a single frontal channel. Clin Neurophysiol. 2020;131(8):1726-7.

36. Murri L, Gori S, Massetani R, Bonanni E, Marcella F, Milani S. Evaluation of acute ischemic stroke using quantitative EEG: a comparison with conventional EEG and CT scan. Neurophysiol Clin. 1998;28(3):249-57.

37. Luu P, Tucker DM, Englander R, Lockfeld A, Lutsep H, Oken B. Localizing acute stroke-related EEG changes: assessing the effects of spatial undersampling. J Clin Neurophysiol. 2001;18(4):302-17.

38. Vespa PM, O'Phelan K, Shah M, Mirabelli J, Starkman S, Kidwell C, et al. Acute seizures after intracerebral hemorrhage: a factor in progressive midline shift and outcome. Neurology. 2003;60(9):1441-6.

39. Wang Y, Zhang X, Huang J, Zhu M, Guan Q, Liu C. Associations between EEG beta power abnormality and diagnosis in cognitive impairment post cerebral infarcts. J Mol Neurosci. 2013;49(3):632-8.

40. Wolf MEE, A.D. Hennerici, M.G. Chatzikonstantinou, A. EEG characteristics of acute ischemic stroke patients without seizures: Generalized but not focal EEG pathology is associated with clinical deterioration. Cerebrovascular Diseases. 2016;41:285

41. Sainio K, Stenberg D, Keskimaki I, Muuronen A, Kaste M. Visual and spectral EEG analysis in the evaluation of the outcome in patients with ischemic brain infarction. Electroencephalogr Clin Neurophysiol. 1983;56(2):117-24.

42. Charlin CT, MCalvet U, Martinez P, Larrue V. The clinical significance of periodic lateralized epileptiform discharges in acute ischemic stroke. J Stroke Cerebrovasc Dis. 2000;9(6):298-302.

43. Cuspineda E, Machado C, Aubert E, Galan L, Llopis F, Avila Y. Predicting outcome in acute stroke: a comparison between QEEG and the Canadian Neurological Scale. Clin Electroencephalogr. 2003;34(1):1-4.

44. Cuspineda E, Machado C, Galan L, Aubert E, Alvarez MA, Llopis F, et al. QEEG prognostic value in acute stroke. Clin EEG Neurosci. 2007;38(3):155-60.

45. Su YY, Wang M, Chen WB, Fu P, Yang QL, Li HL, et al. Early prediction of poor outcome in severe hemispheric stroke by EEG patterns and gradings. Neurol Res. 2013;35(5):512-6.

46. Lima FO, Ricardo JAG, Coan AC, Soriano DC, Avelar WM, Min LL. Electroencephalography Patterns and Prognosis in Acute Ischemic Stroke. Cerebrovasc Dis. 2017:44(3-4):128-34.

47. Bentes C, Peralta AR, Martins H, Casimiro C, Morgado C, Franco AC, et al. Seizures, electroencephalographic abnormalities, and outcome of ischemic stroke patients. Epilepsia Open. 2017;2(4):441-52.

48. Xin X, Chang J, Gao Y, Shi Y. Correlation Between the Revised Brain Symmetry Index, an EEG Feature Index, and Short-term Prognosis in Acute Ischemic Stroke. J Clin Neurophysiol. 2017;34(2):162-7.

49. Bentes C, Peralta AR, Viana P, Martins H, Morgado C, Casimiro C, et al. Quantitative EEG and functional outcome following acute ischemic stroke. Clin Neurophysiol. 2018;129(8):1680-7.

50. Kuznietsov AA. Prediction of the functional outcome of cerebral ischemic supratentorial stroke acute period on the basis of spectral analysis of the brain bioelectrical activity. Zaporozhye Med J. 2018;20:324-9.

51. Rogers J, Middleton S, Wilson PH, Johnstone SJ. Predicting functional outcomes after stroke: an observational study of acute single-channel EEG. Topics Stroke Rehabil. 2020;27(3):161-72. 
52. Juhasz C, Kamondi A, Szirmai I. Spectral EEG analysis following hemispheric stroke: evidences of transhemispheric diaschisis. Acta Neurol Scand. 1997:96(6):397-400.

53. Finnigan SP, Walsh M, Rose SE, Chalk JB. Quantitative EEG indices of subacute ischaemic stroke correlate with clinical outcomes. Clin Neurophysiol. 2007;1 18(11):2525-32.

54. De Herdt VDB, E. Janssen, H.: Hodl, S. Hemelsoet, D. Boon, P. Epileptic discharges in the acute phase of stroke. Eur Stroke J. 2018;3:397.

55. Gur AY, Neufeld MY, Treves TA, Aronovich BD, Bornstein NM, Korczyn AD. EEG as predictor of dementia following first ischemic stroke. Acta Neurol Scand. 1994;90(4):263-5.

56. Song Y, Zang DW, Jin YY, Wang ZJ, Ni HY, Yin JZ, et al. Background rhythm frequency and theta power of quantitative EEG analysis: predictive biomarkers for cognitive impairment post-cerebral infarcts. Clin EEG Neurosc. 2015;46(2):142-6.

57. Jiang M, Su Y, Liu G, Chen W, Gao D. Predicting the non-survival outcome of large hemispheric infarction patients via quantitative electroencephalography: Superiority to visual electroencephalography and the Glasgow Coma Scale. Neurosci Lett. 2019;706:88-92.

58. Bentes C, Martins H, Peralta AR, Morgado C, Casimiro C, Franco AC, et al. Early EEG predicts poststroke epilepsy. Epilepsia Open. 2018;3(2):203-12.

59. Andersen KK, Olsen TS, Dehlendorff C, LP K. Hemorrhagic and ischemic strokes compared: stroke severity, mortality, and risk factors. Stroke. 2019;40(6):2068-72.

60. Sergot PB, Maza AJ, Derrick BJ, Smith LM, Berti LT, Wilcox MR, et al. Portable Neuromonitoring Device Detects Large Vessel Occlusion in Suspected Acute Ischemic Stroke. Stroke. 2021;52(4):1437-40.

61. Boers AM, Jansen IG, Beenen LF, Devlin TG, San Roman L, Heo JH, et al. Association of follow-up infarct volume with functional outcome in acute ischemic stroke: a pooled analysis of seven randomized trials Journal of neurointerventional surgery. 2018:10(12):1137-42.

62. Stinear CM, Byblow WD, Ackerley SJ, Smith MC, Borges VM, PA B. PREP2: A biomarker-based algorithm for predicting upper limb function after stroke. Ann Clin Transl Neurol. 2017:4(11):811-20.

63. Smith MC, Barber PA, CM S. The TWIST algorithm predicts time to walking independently after stroke. Neurorehabil Neural Repair. 2017;31:955-64.

64. Stinear CM, Smith MC, WD B. Prediction Tools for Stroke Rehabilitation. Stroke. 2019;50(11):3314-22.

65. Tang EY, Price Cl, Robinson LCE. (STROKOG) obotSaCC. Assessing the Predictive Validity of Simple Dementia Risk Models in Harmonised Stroke Cohorts. Stroke. 2020;51:2095-102.

66. Kalaria RN, Akinyemi R. M I Stroke injury, cognitive impairment and vascular dementia. Biochim Biophys Acta. 2016;1862(915):25.

67. van Meenen LCC, van Stigt MN, Siegers A, Smeekes MD, van Grondelle JAF, Geuzebroek G, et al. Detection of Large Vessel Occlusion Stroke in the Prehospital Setting: Electroencephalography as a Potential Triage Instrument. Stroke. 2021;52(7):e347-55.

68. Goyal M, Ospel JM, Kim BJ, Kashani N, Duvekot MH, Roozenbeek B, et al. A Bayesian Framework to Optimize Performance of Pre-Hospital Stroke Triage Scales. J Stroke. 2021;23(3):443.

\section{Publisher's Note}

Springer Nature remains neutral with regard to jurisdictional claims in published maps and institutional affiliations.

Ready to submit your research? Choose BMC and benefit from:

- fast, convenient online submission

- thorough peer review by experienced researchers in your field

- rapid publication on acceptance

- support for research data, including large and complex data types

- gold Open Access which fosters wider collaboration and increased citations

- maximum visibility for your research: over $100 \mathrm{M}$ website views per year

At $\mathrm{BMC}$, research is always in progress.

Learn more biomedcentral.com/submissions 